\title{
Deformation Study of Papandayan Volcano using GPS Survey Method and Its Correlation with Seismic Data Observation
}

\author{
Dina A. Sarsito ${ }^{1}$, Heri Andreas ${ }^{1}$, Hasanuddin Z. Abidin ${ }^{1}$, Muhammad Gamal ${ }^{1}$, \\ Ony K. Suganda ${ }^{2} \&$ Muhammad Hendrasto $^{2}$ \\ ${ }^{1}$ Department of Geodetic Engineering, Institute of Technology Bandung (ITB), Jl. \\ Ganesha 10, Bandung 40132, INDONESIA. e-mail: dsarsito@gd.itb.ac.id Tel: 62-22- \\ 2534286; Fax: 62-22-2534286. \\ ${ }^{2}$ Directorate of Vulcanology and Geological Hazard Mitigation (DVGHM), Jl. \\ Diponegoro 57, Bandung 40132, INDONESIA
}

\begin{abstract}
Papandayan volcano located in the southern part of Garut regency, around $70 \mathrm{~km}$ away from Bandung city, West Java. Many methods carried out to monitoring the activities of volcano, both continuously or periodically, one of the monitoring method is periodically GPS survey. Basically those surveys are carried out to understand the pattern and velocity of displacement which occurred in the volcano body, both horizontally and vertically, and also others deformation elements such as; translation, rotation and dilatation. The Mogi modeling was also used to determine the location and volume of the pressure source which caused deformation of volcano body. By comparing seismic activity and the deformation reveal from GPS measurement, before, during and after eruption, it could be understood there is a correlation between the seismicity and its deformation. These studies is hoping that GPS measurement in Papandayan volcano could be one of supported method to determine the volcano activities, at least in Papandayan volcano.
\end{abstract}

Keywords: Deformation; GPS; Papandayan; Volcano.

\section{Introduction}

Indonesia is located in three subduction plate intersection, such as; Eurasian plate, Pacific plate, and Indian-Australian plate where they move with the different direction. This matter caused the occurrences of volcanic ridge formed during million years along Indonesian archipelago.

Based on VSI data (VSI, 1995), Indonesia has 129 of active volcanoes (Table 1) with 271 eruption points as a consequence of the interaction and collision between several continent plates. With 200 million of dense population and Java island is the most dense population, here we could not denied that volcanic hazards is a real thing for Indonesian people. VSI said about $10 \%$ of Indonesian citizen lived in dangerous area of volcanic eruption, here needs an efforts to

Received April 2006, Revised September $14^{\text {th }}$ 2006, Accepted for publication September $14^{\text {th }} 2006$. 
reduce the negative impact which may happen by monitoring of volcanic activities. On the other hands monitoring of Indonesian volcanoes must be done maximally and continuously. This research carried out by joint cooperation between Study Group of Geodesy, Bandung Institute of Technology (ITB) and Center of Volcanology and Geological Hazards Mitigation, Bandung.

Table 1 Distribution and type of Indonesian volcano [VSI, 1995].

\begin{tabular}{|c|c|c|c|c|}
\hline \multirow{2}{*}{ Island/Area } & \multicolumn{3}{|c|}{ Total Volcanoes } & \multirow[b]{2}{*}{ Tot a I } \\
\hline & A type & B type & C type & \\
\hline Sumatera & 12 & 12 & 6 & 30 \\
\hline Jawa & 21 & 8 & 5 & 34 \\
\hline Bali & 2 & - & - & 2 \\
\hline Nusa Tenggara & 20 & 3 & 5 & 28 \\
\hline Laut Banda & 9 & 1 & - & 10 \\
\hline Sulawesi Utara & 6 & 2 & 5 & 13 \\
\hline \multirow{2}{*}{$\begin{array}{l}\text { Sanghe } \\
\text { Halmahera }\end{array}$} & 5 & - & - & 5 \\
\hline & 5 & 2 & - & 7 \\
\hline Total & 80 & 28 & 21 & 129 \\
\hline \multirow{2}{*}{\multicolumn{5}{|c|}{$\begin{array}{ll}\text { Type-A : } & \text { Volcanoes which have m agmatic or phreatic eruption and recorded sinoe } 1600 . \\
\text { Type-B : } & \text { Volcanoes which have no magmatic or semi-m agm atic activities since } 1600, \\
& \text { but they show fumarol a and solfatara activities. }\end{array}$}} \\
\hline & & & fum arola & ara activities. \\
\hline
\end{tabular}

\section{Papandayan Volcano}

Research object is Papandayan volcano as one of the active volcano in Indonesia, located in West Java province, Garut regency and geographical position $7^{\circ} 19^{\prime}$ latitude and $107^{\circ} 44^{\prime}$ longitude, altitude $2662 \mathrm{~m}$ above sea level as an A type volcano, Figure 1.
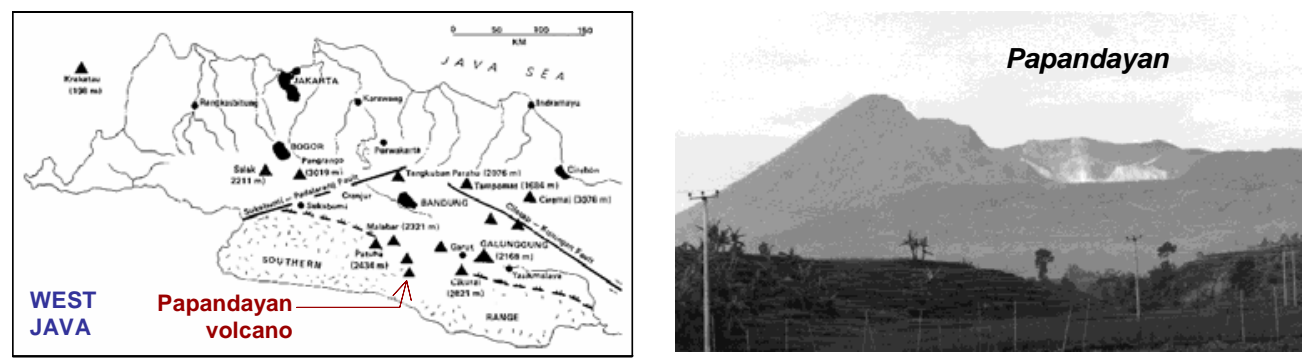

Figure 1 Location of Papandayan volcano. 
Historical eruption of Papandayan volcano as follow:

1772 In the night at August 11-12, large eruptions occurred from central crater and surge flowing down killed 2951 people and destroy 40 villages.

1882 On May 28, in the nice evening, at Campaka Warna thunders audible presume from inside of Papandayan volcano.

1923 March 11, eruption occurred, discharging mud and stones for 150 $\mathrm{m}$ distance. There are 7 eruptions inner Kawah Baru and the eruption starting by feel earthquake in Cisurupan.

1924 January 25, temperature of Kawah Mas increased from 364oC to $500 \circ C$, then eruption occurred in Kawah Mas and kawah baru, the forrest destroyed by mud and stones, eruption material fall at eastern part almost reached Cisurupan.

1925 February 21, mud eruption occurred at Kawah Nangklak strong gas discharge and mud rain.

1926 At Kawah Mas, small mix mud and sulphur eruption occurred. In Kawah Baru strong wind blows flew away sulphur powder reach $300 \mathrm{~m}$ long the north-east and to the south-west reached $100 \mathrm{~m}$ long ending by mud-sulphur eruption.

1927 Februari 16-18, increasing of seismicities occurred at Kawah Mas and until now fumarolic and solfataric steam still occurred accompanied by spouted hot water.

1942 August 15-16, occurred new eruption hole/crater.

1993 July 17, mud explosion occurred in Kawah Baru.

1998 On June, volcanic activities increased initially by increasing of seismicity, mud and gas discharge occurred at fumarolic vents such as Kawah Mas, about $5 \mathrm{~m}$.

2002 Starting on November 11 strong increasing of activities occurred, on November 13-20 large eruption and directed blast occurred, activities decreased on December 21, landslide occurred at Kawah Nangklak flank and caused flood along Cibeureum gede river reached $7 \mathrm{~km}$ to Cimanuk river sink several houses and caused big erosion along the river.

\section{GPS Survey and Seismic Observation in Papandayan}

Until now GPS campaign in Papandayan volcano has carried out for 8 campaigns, as show on Table 2. During the last campaign there are 7 new benchmark installed; CLLY, KMA1 (substitute the lost KMAS benchmark), NGL1 (substitute the lost NGLK benchmark), ALUN, CSRN, SEIS (fix seismogram coordinate) and WNGR (Figure 2), the aim of this addition to substitute the lost former benchmark which lost caused by the November 2002 
eruption. All campaigns carried out by using Geodetic GPS receiver dualfrequency such as; Ashtech Z-XII3, Leica System 300 and Leica System 500, duration of measurement 8 to 16 hours.

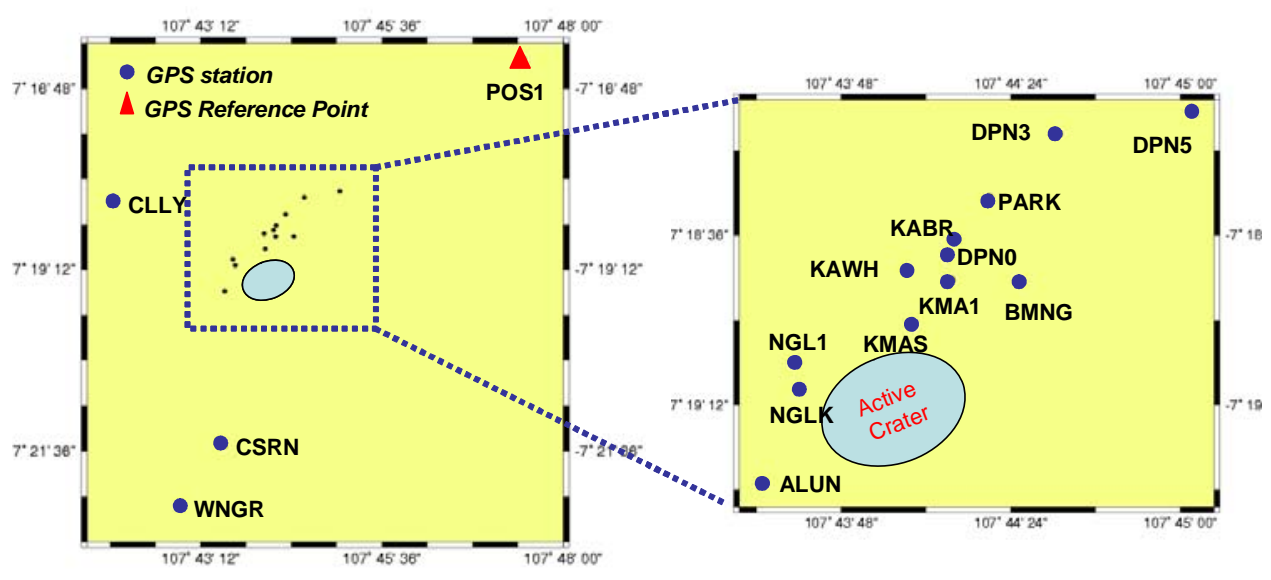

Figure 2 GPS stations in Papandayan volcano.

Seismic data of 1998 - 2005 is incomplete caused the obstacle on determination of cumulative energy which happens during the all 8 GPS campaign (1998 2005) and also seismic data derived only from one component seismic station, thus hypocenter not determined.

Table 2 Papandayan GPS survey.

\begin{tabular}{cll}
\hline GPS Survey & \multicolumn{1}{c}{ Time Survey } & \multicolumn{1}{c}{ Observation Station } \\
\hline Survey - 1 & 22-23 Oct. 1998 & POS1, NGLK, KAWH, PARK, BMNG \\
Survey - 2 & 25-26 Mar. 1999 & POS1, NGLK, KAWH, PARK, BMNG \\
Survey - 3 & 23-24 Des. 1999 & POS1, NGLK, KAWH, PARK, BMNG \\
Survey - 4 & 23-24 Jun. 2001 & POS1, NGLK, KAWH, PARK, BMNG \\
Survey - 5 & 11-12 Aug. 2002 & POS1, NGLK, KAWH, PARK, BMNG, \\
& & DPN0, DPN3, DPN5, KMAS \\
Survey - 6 & $\begin{array}{l}\text { 13-15 Nov. 2002 } \\
\text { 17 Nov. 2002 }\end{array}$ & POS1, PARK, DPN0, DPN3, DPN5 \\
& $\begin{array}{l}\text { 18 Nov. 2002 } \\
\text { 19 Nov. 2002 }\end{array}$ & POS1,DPN0, KMAS, KWAH \\
& 9-10 Jun. 2003 & POS1, KAWH, PARK, BMNG, DPN3, DPN5, \\
Survey - 7 & KWAH2 \\
Survey - 8 & 14-17 Aug. 2005 & POS1, KAWH, PARK, BMNG, DPN3, DPN5, \\
& & KABR, CLLY, KMA1, NGLK1, ALUN, CSRN, \\
& & WNGR, SEIS \\
\hline
\end{tabular}


Theoretically, deformation and volcanic seismicity are correlated, however the characteristic and the correlation pattern will be different depend on geological structure of the volcano and the internal system of magma vent. Then, correlation of both methods in this volcano monitoring will studied, applied, analyzed and tested.

GPS survey method give deformation signal of volcano body in 3D geocentric coordinate system from representative points by flexible interval time. Volcano deformation could be an inflation or deflation. Inflation caused by magma movement to the surface and deflation occurred when magma reach the surface and flowing down together with the energy released, this magma movement will shaking volcano body where this case will record by seismogram surrounding the volcano. On the other hand the deformation of magma body and seismic activity will positively correlate (Table 3).

Table 3 Correlation of volcano deformation and seismic activity.

\begin{tabular}{c|c|c|c}
\cline { 2 - 3 } & GPS & Seismogram & \\
\hline Magma Activity & Volcano deformation & Seismic Activities & Correlation \\
\hline Magma Movement & Inflation & Large & Positive \\
Magma Discharge & Deflation & Small & Positive \\
\hline
\end{tabular}

\section{$4 \quad$ Data Processing and Result}

GPS data processing carried out by using scientific software BERNESSE 4.2 [2]. Deviations standard of coordinate component, show on Figure 3. Horizontal component (easting and northing) have $4 \mathrm{~mm}$ deviation standard, generally in level 1-2 mm. Ellipsoid height component around $7 \mathrm{~mm}$, generally 2-5 mm.

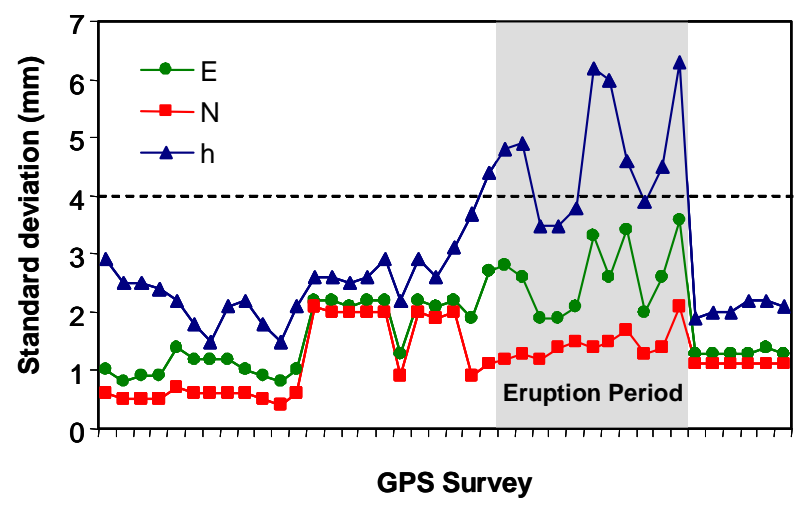

Figure 3 Coordinat deviation standard of 8 surveys. 


\subsection{GPS Coordinate Points}

Geodetic coordinate (latitude, longitude, ellipsoid height) of GPS network points with their deviation standard which revealed by GPS processing data show on Table 4. Noticed that 5 campaigns carried out during the November 2002 eruptions, and 8 campaigns carried out 22 months afterward with the aim is to understand the deformation status of Papandayan volcano and the correlation with the seismicity.

\subsection{Baseline and Coordinate Changes}

By 8 GPS campaign data revealed both horizontal and vertical coordinate as show Figures 4, 5, 6a, and 6b. Changes in whole campaign occurred periodically, where the whole volcano body increased or decreased vertically and move to the northeast and southwest horizontally.

Table 4 GPS points network coordinate of Papandayan volcano.

\begin{tabular}{|c|c|c|c|c|c|c|}
\hline \multicolumn{7}{|c|}{ GPS OBSERVATION RESULTS ( EPOCH 1) } \\
\hline Station & Longitude & $\neg L(\mathrm{~m})$ & Latitude & $\neg \mathrm{B}(\mathrm{m})$ & $\mathrm{h}$ Ellipsoid & $\neg \mathrm{h}$ Ellip(m) \\
\hline POS1 & $7^{\circ} 16^{\prime} 24.26048^{\prime \prime} \mathrm{S}$ & 0.0000 & $107^{\circ} 47^{\prime} 28.74811^{\prime \prime} \mathrm{E}$ & 0.0000 & 1148.4904 & 0.0000 \\
\hline NGLK & $7^{\circ} 19^{\prime} 08.46359^{\prime \prime} \mathrm{S}$ & 0.0006 & $107^{\circ} 43^{\prime} 39.41730^{\prime \prime} \mathrm{E}$ & 0.0010 & 2426.7686 & 0.0022 \\
\hline KAWH & $7^{\circ} 18^{\prime} 43.07654^{\prime \prime} \mathrm{S}$ & 0.0005 & $107^{\circ} 44^{\prime} 02.34135^{\prime \prime} \mathrm{E}$ & 0.0008 & 2197.6694 & 0.0017 \\
\hline PARK & $7^{\circ} 18^{\prime} 27.82112^{\prime \prime} \mathrm{S}$ & 0.0005 & $107^{\circ} 44^{\prime} 19.49601^{\prime \prime} \mathrm{E}$ & 0.0009 & 2027.8624 & 0.0019 \\
\hline BMNG & $7^{\circ} 18^{\prime} 45.32883^{\prime \prime} \mathrm{S}$ & 0.0005 & $107^{\circ} 44^{\prime} 26.05085^{\prime \prime} \mathrm{E}$ & 0.0009 & 2124.6565 & 0.0018 \\
\hline
\end{tabular}

\begin{tabular}{|c|c|c|c|c|c|c|}
\hline \multicolumn{7}{|c|}{ GPS OBSERVATION RESULTS ( EPOCH 2) } \\
\hline Station & Longitude & $\neg$ L(m) & Latitude & $\neg \mathrm{B}(\mathrm{m})$ & $\mathrm{h}$ Ellipsoid & $\neg \mathrm{h}$ Ellip(m) \\
\hline POS1 & $7^{\circ} 16^{\prime} 24.26048^{\prime \prime} \mathrm{S}$ & 0.0000 & $107^{\circ} 47^{\prime} 28.74811^{\prime \prime} \mathrm{E}$ & 0.0000 & 1148.4904 & 0.0000 \\
\hline NGLK & $7^{\circ} 19^{\prime} 08.46319^{\prime \prime} \mathrm{S}$ & 0.0007 & $107^{\circ} 43^{\prime} 39.41783^{\prime \prime} \mathrm{E}$ & 0.0014 & 2426.7075 & 0.0029 \\
\hline KAWH & $7^{\circ} 18^{\prime} 43.07635^{\prime \prime} \mathrm{S}$ & 0.0006 & $107^{\circ} 44^{\prime} 02.34174^{\prime \prime} \mathrm{E}$ & 0.0012 & 2197.6417 & 0.0025 \\
\hline PARK & $7^{\circ} 18^{\prime} 27.82085^{\prime \prime} \mathrm{S}$ & 0.0006 & $107^{\circ} 44^{\prime} 19.49624^{\prime \prime} \mathrm{E}$ & 0.0012 & 2027.8874 & 0.0025 \\
\hline BMNG & $7^{\circ} 18^{\prime} 45.32835^{\prime \prime} \mathrm{S}$ & 0.0006 & $107^{\circ} 44^{\prime} 26.05112^{\prime \prime} \mathrm{E}$ & 0.0012 & 2124.6844 & 0.0024 \\
\hline
\end{tabular}

\begin{tabular}{|c|c|c|c|c|c|c|}
\hline \multicolumn{7}{|c|}{ GPS OBSERVATION RESULTS ( EPOCH 3) } \\
\hline Station & Longitude & $\neg$ L(m) & Latitude & $\neg \mathrm{B}(\mathrm{m})$ & $\mathrm{h}$ Ellipsoid & $\neg \mathrm{h}$ Ellip(m) \\
\hline POS1 & $7^{\circ} 16^{\prime} 24.26048^{\prime \prime} \mathrm{S}$ & 0.0000 & $107^{\circ} 47^{\prime} 28.74811^{\prime \prime} \mathrm{E}$ & 0.0000 & 1148.4904 & 0.0000 \\
\hline NGLK & $7^{\circ} 19^{\prime} 08.46291^{\prime \prime} \mathrm{S}$ & 0.0005 & $107^{\circ} 43^{\prime} 39.41747^{\prime \prime} \mathrm{E}$ & 0.0011 & 2426.7620 & 0.0018 \\
\hline KAWH & $7^{\circ} 18^{\prime} 43.07648^{\prime \prime} \mathrm{S}$ & 0.0006 & $107^{\circ} 44^{\prime} 02.34191^{\prime \prime} \mathrm{E}$ & 0.0011 & 2197.6922 & 0.0024 \\
\hline PARK & $7^{\circ} 18^{\prime} 27.82111^{\prime \prime} \mathrm{S}$ & 0.0005 & $107^{\circ} 44^{\prime} 19.49593^{\prime \prime} \mathrm{E}$ & 0.0009 & 2027.8890 & 0.0018 \\
\hline BMNG & $7^{\circ} 18^{\prime} 45.32865^{\prime \prime} \mathrm{S}$ & 0.0005 & $107^{\circ} 44^{\prime} 26.05106^{\prime \prime} \mathrm{E}$ & 0.0009 & 2124.6697 & 0.0019 \\
\hline
\end{tabular}

\begin{tabular}{|c|c|c|c|c|c|c|}
\hline \multicolumn{7}{|c|}{ GPS OBSERVATION RESULTS ( EPOCH 4) } \\
\hline Station & Longitude & $\neg$ L $(\mathrm{m})$ & Latitude & $\neg \mathrm{B}(\mathrm{m})$ & $\mathrm{h}$ Ellipsoid & $\neg \mathrm{h}$ Ellip(m) \\
\hline POS1 & $7^{\circ} 16^{\prime} 24.26048^{\prime \prime} \mathrm{S}$ & 0.0000 & $107^{\circ} 47^{\prime} 28.74811^{\prime \prime} \mathrm{E}$ & 0.0000 & 1148.4904 & 0.0000 \\
\hline NGLK & $7^{\circ} 19^{\prime} 08.46238^{\prime \prime} \mathrm{S}$ & 0.0021 & $107^{\circ} 43^{\prime} 39.41749^{\prime \prime} \mathrm{E}$ & 0.0022 & 2426.6571 & 0.0026 \\
\hline KAWH & $7^{\circ} 18^{\prime} 43.07606^{\prime \prime} \mathrm{S}$ & 0.002 & $107^{\circ} 44^{\prime} 02.34162^{\prime \prime} \mathrm{E}$ & 0.0022 & 2197.6577 & 0.0026 \\
\hline PARK & $7^{\circ} 18^{\prime} 27.82107^{\prime \prime} \mathrm{S}$ & 0.002 & $107^{\circ} 44^{\prime} 19.49624^{\prime \prime} \mathrm{E}$ & 0.0021 & 2027.9184 & 0.0025 \\
\hline BMNG & $7^{\circ} 18^{\prime} 45.32843^{\prime \prime} \mathrm{S}$ & 0.002 & $107^{\circ} 44^{\prime} 26.05105^{\prime \prime} \mathrm{E}$ & 0.0022 & 2124.6733 & 0.0026 \\
\hline
\end{tabular}


Tabel 4 GPS points network coordinate of Papandayan volcano. (continue)

\begin{tabular}{|c|c|c|c|c|c|c|}
\hline \multicolumn{7}{|c|}{ GPS OBSERVATION RESULTS ( EPOCH 5) } \\
\hline Station & Longitude & $\neg$ L(m) & Latitude & $\neg \mathrm{B}(\mathrm{m})$ & $\mathrm{h}$ Ellipsoid & $\neg \mathrm{h}$ Ellip(m) \\
\hline POS1 & $7^{\circ} 16^{\prime} 24.26048^{\prime \prime} \mathrm{S}$ & 0.0000 & $107^{\circ} 47^{\prime} 28.74811^{\prime \prime} \mathrm{E}$ & 0.0000 & 1148.4904 & 0.0000 \\
\hline NGLK & $7^{\circ} 19^{\prime} 08.46237^{\prime \prime} \mathrm{S}$ & 0.0020 & $107^{\circ} 43^{\prime} 39.41722^{\prime \prime} \mathrm{E}$ & 0.0022 & 2426.6947 & 0.0029 \\
\hline KMAS & $7^{\circ} 18^{\prime} 55.28806^{\prime \prime} \mathrm{S}$ & 0.0009 & $107^{\circ} 44^{\prime} 03.32420^{\prime \prime} \mathrm{E}$ & 0.0013 & 2161.2822 & 0.0022 \\
\hline KAWH & $7^{\circ} 18^{\prime} 43.07618^{\prime \prime} \mathrm{S}$ & 0.0019 & $107^{\circ} 44^{\prime} 02.34155^{\prime \prime} \mathrm{E}$ & 0.0025 & 2197.6534 & 0.0034 \\
\hline PARK & $7^{\circ} 18^{\prime} 2.82124^{\prime \prime} \mathrm{S}$ & 0.0019 & $107^{\circ} 44^{\prime} 19.49651^{\prime \prime} \mathrm{E}$ & 0.0023 & 2027.9168 & 0.0027 \\
\hline BMNG & $7^{\circ} 18^{\prime} 45.32789^{\prime \prime} \mathrm{S}$ & 0.0020 & $107^{\circ} 44^{\prime} 26.05193^{\prime \prime} \mathrm{E}$ & 0.0032 & 2124.5829 & 0.0035 \\
\hline DPN0 & $7^{\circ} 18^{\prime} 40.57983^{\prime \prime} \mathrm{S}$ & 0.0009 & $107^{\circ} 44^{\prime} 09.98676 " \mathrm{E}$ & 0.0019 & 2074.2173 & 0.0037 \\
\hline DPN3 & $7^{\circ} 18^{\prime} 14.41003^{\prime \prime} \mathrm{S}$ & 0.0011 & $107^{\circ} 44^{\prime} 34.25790^{\prime \prime} \mathrm{E}$ & 0.0027 & 1943.3206 & 0.0044 \\
\hline DPN5 & $7^{\circ} 18^{\prime} 09.64556^{\prime \prime} \mathrm{S}$ & 0.0012 & $107^{\circ} 45^{\prime} 02.59428^{\prime \prime} \mathrm{E}$ & 0.0028 & 1804.5354 & 0.0048 \\
\hline
\end{tabular}

\begin{tabular}{|c|c|c|c|c|c|c|}
\hline \multicolumn{7}{|c|}{ GPS OBSERVATION RESULTS (EPOCH 6) } \\
\hline Station & Longitude & $\neg \mathrm{L}(\mathrm{m})$ & $\begin{array}{l}\text { Latitude } \\
\end{array}$ & $\neg \mathrm{B}(\mathrm{m})$ & h Ellipsoid & $\neg \mathrm{h}$ Ellip $(\mathrm{m})$ \\
\hline POS1 & $7^{\circ} 16^{\prime} 24.26048^{\prime \prime} \mathrm{S}$ & 0.0000 & $107^{\circ} 47^{\prime} 28.74811^{\prime \prime} \mathrm{E}$ & 0.0000 & 1148.4904 & 0.0000 \\
\hline KMAS (18NOV) & $7^{\circ} 18^{\prime} 55.28581^{\prime \prime} \mathrm{S}$ & 0.0013 & $107^{\circ} 44^{\prime} 03.32767^{\prime \prime} \mathrm{E}$ & 0.0026 & 2161.647 & 0.0049 \\
\hline KAWH (18NOV) & $7^{\circ} 18^{\prime} 43.07061^{\prime \prime} \mathrm{S}$ & 0.0012 & $107^{\circ} 44^{\prime} 02.34506^{\prime \prime} \mathrm{E}$ & 0.0019 & 2197.9255 & 0.0035 \\
\hline PARK (15NOV) & $7^{\circ} 18^{\prime} 27.82078^{\prime \prime} \mathrm{S}$ & 0.0014 & $107^{\circ} 44^{\prime} 19.49631^{\prime \prime} \mathrm{E}$ & 0.0019 & 2028.0189 & 0.0035 \\
\hline PARK (17NOV) & $7^{\circ} 18^{\prime} 27.82086^{\prime \prime} \mathrm{S}$ & 0.0015 & $107^{\circ} 44^{\prime} 19.49636^{\prime \prime} \mathrm{E}$ & 0.0021 & 2027.9813 & 0.0038 \\
\hline BMNG (19NOV) & $7^{\circ} 18^{\prime} 45.32866^{\prime \prime} \mathrm{S}$ & 0.0014 & $107^{\circ} 44^{\prime} 26.05065^{\prime \prime} \mathrm{E}$ & 0.0033 & 2124.7393 & 0.0062 \\
\hline DPNO (13NOV) & $7^{\circ} 18^{\prime} 40.57682^{\prime \prime} \mathrm{S}$ & 0.0015 & $107^{\circ} 44^{\prime} 09.99020^{\prime \prime} \mathrm{E}$ & 0.0026 & 2074.5039 & 0.0060 \\
\hline DPNO (17NOV) & $7^{\circ} 18^{\prime} 40.57704^{\prime \prime} \mathrm{S}$ & 0.0017 & $107^{\circ} 44^{\prime} 09.98988^{\prime \prime} \mathrm{E}$ & 0.0034 & 2074.5019 & 0.0046 \\
\hline DPNO (18NOV & $7^{\circ} 18^{\prime} 40.57708^{\prime \prime} \mathrm{S}$ & 0.0013 & $107^{\circ} 44^{\prime} 09.98999^{\prime \prime} \mathrm{E}$ & 0.0020 & 2074.4946 & 0.0039 \\
\hline DPN3 (14NOV) & $7^{\circ} 18^{\prime} 14.41050^{\prime \prime} \mathrm{S}$ & 0.0036 & $107^{\circ} 44^{\prime} 34.25611^{\prime \prime} \mathrm{E}$ & 0.0076 & 1943.4169 & 0.0126 \\
\hline DPN3 (15NOV) & $7^{\circ} 18^{\prime} 14.40982^{\prime \prime} \mathrm{S}$ & 0.0014 & $107^{\circ} 44^{\prime} 34.25649^{\prime \prime} \mathrm{E}$ & 0.0026 & 1943.3937 & 0.0045 \\
\hline DPN5 (14NOV) & $7^{\circ} 18^{\prime} 09.64568^{\prime \prime} \mathrm{S}$ & 0.0021 & $107^{\circ} 45^{\prime} 02.59342^{\prime \prime} \mathrm{E}$ & 0.0036 & 1804.6394 & 0.0063 \\
\hline
\end{tabular}

\begin{tabular}{|c|c|c|c|c|c|c|}
\hline \multicolumn{7}{|c|}{ GPS OBSERVATION RESULTS (EPOCH 7) } \\
\hline Station & Longitude & $\neg \mathrm{L}(\mathrm{m})$ & Latitude & $\neg \mathrm{B}(\mathrm{m})$ & $\mathrm{h}$ Ellipsoid & ᄀ $\mathrm{h}$ Ellip $(\mathrm{m})$ \\
\hline POS1 & $7^{\circ} 16^{\prime} 24.26048^{\prime \prime} \mathrm{S}$ & 0.0000 & $107^{\circ} 47^{\prime} 28.74811^{\prime \prime E}$ & 0.0000 & 1148.4904 & 0.0000 \\
\hline KAWH & $7^{\circ} 18^{\prime} 43.07200^{\prime \prime} \mathrm{S}$ & 0.0011 & $107^{\circ} 44^{\prime} 02.34397^{\prime \prime} \mathrm{E}$ & 0.0013 & 2197.8055 & 0.0019 \\
\hline PARK & $7^{\circ} 18^{\prime} 27.82135^{\prime \prime} \mathrm{S}$ & 0.0011 & $107^{\circ} 44^{\prime} 19.49593^{\prime \prime} \mathrm{E}$ & 0.0013 & 2027.9122 & 0.0020 \\
\hline BMNG & $7^{\circ} 18^{\prime} 45.32835^{\prime \prime} \mathrm{S}$ & 0.0011 & $107^{\circ} 44^{\prime} 26.04972^{\prime \prime} \mathrm{E}$ & 0.0013 & 2124.6007 & 0.0020 \\
\hline DPN3 & $7^{\circ} 18^{\prime} 14.40972^{\prime \prime} \mathrm{S}$ & 0.0011 & $107^{\circ} 44^{\prime} 34.25626^{\prime \prime} \mathrm{E}$ & 0.0013 & 1943.2771 & 0.0022 \\
\hline DPN5 & $7^{\circ} 18^{\prime} 09.64536^{\prime \prime} \mathrm{S}$ & 0.0011 & $107^{\circ} 45^{\prime} 02.59322^{\prime \prime} \mathrm{E}$ & 0.0014 & 1804.5497 & 0.0022 \\
\hline KABR & $7^{\circ} 18^{\prime} 36.72898^{\prime \prime} \mathrm{S}$ & 0.0011 & $107^{\circ} 44^{\prime} 11.89710^{\prime \prime} \mathrm{E}$ & 0.0013 & 2061.7172 & 0.0021 \\
\hline
\end{tabular}

\begin{tabular}{|c|c|c|c|c|c|c|}
\hline \multicolumn{7}{|c|}{ GPS OBSERVATION RESULTS (EPOCH 8) } \\
\hline Station & Longitude & $\neg \mathrm{L}(\mathrm{m})$ & Latitude & $\neg \mathrm{B}(\mathrm{m})$ & $\mathrm{h}$ Ellipsoid & $\neg \mathrm{h}$ Ellip $(\mathrm{m})$ \\
\hline POS1 & $7^{\circ} 16^{\prime} 24.26048^{\prime \prime} \mathrm{S}$ & 0.0000 & $107^{\circ} 47^{\prime} 28.74811^{\prime \prime} \mathrm{E}$ & 0.0000 & 1148.4904 & 0.0000 \\
\hline KAWH & $7^{\circ} 18^{\prime} 43.07390^{\prime \prime} \mathrm{S}$ & 0.0006 & $107^{\circ} 44^{\prime} 02.34393^{\prime \prime} \mathrm{E}$ & 0.0012 & 2197.6808 & 0.0024 \\
\hline PARK & $7^{\circ} 18^{\prime} 27.82187^{\prime \prime} \mathrm{S}$ & 0.0017 & $107^{\circ} 44^{\prime} 19.49541^{\prime \prime} \mathrm{E}$ & 0.0019 & 2027.8500 & 0.0024 \\
\hline BMNG & $7^{\circ} 18^{\prime} 45.32913^{\prime \prime} \mathrm{S}$ & 0.0025 & $107^{\circ} 44^{\prime} 26.04843^{\prime \prime} \mathrm{E}$ & 0.0039 & 2124.5931 & 0.0063 \\
\hline DPN3 & $7^{\circ} 18^{\prime} 14.41043^{\prime \prime} \mathrm{S}$ & 0.0016 & $107^{\circ} 44^{\prime} 34.25613^{\prime \prime} \mathrm{E}$ & 0.002 & 1943.2890 & 0.0027 \\
\hline KABR & $7^{\circ} 18^{\prime} 36.73038^{\prime \prime} \mathrm{S}$ & 0.0014 & $107^{\circ} 44^{\prime} 11.89688^{\prime \prime} \mathrm{E}$ & 0.0016 & 2061.6203 & 0.0021 \\
\hline CLLY & $7^{\circ} 18^{\prime} 16.41497^{\prime \prime} \mathrm{S}$ & 0.0022 & $107^{\circ} 42^{\prime} 00.28281^{\prime \prime} \mathrm{E}$ & 0.0028 & 1916.5611 & 0.0038 \\
\hline KMA1 & $7^{\circ} 18^{\prime} 45.47990^{\prime \prime} \mathrm{S}$ & 0.0014 & $107^{\circ} 44^{\prime} 11.52000^{\prime \prime} \mathrm{E}$ & 0.0016 & 2094.5869 & 0.0020 \\
\hline NGL1 & $7^{\circ} 19^{\prime} 03.39927^{\prime \prime S ~ S ~}$ & 0.0015 & $107^{\circ} 43^{\prime} 37.68740^{\prime \prime} \mathrm{E}$ & 0.0017 & 2351.1070 & 0.0021 \\
\hline ALUN & $7^{\circ} 19^{\prime} 28.90475^{\prime \prime} \mathrm{S}$ & 0.0015 & $107^{\circ} 43^{\prime} 31.20624^{\prime \prime} \mathrm{E}$ & 0.0018 & 2528.7556 & 0.0022 \\
\hline CSRN & $7^{\circ} 21^{\prime} 31.37560^{\prime \prime} \mathrm{S}$ & 0.0016 & $107^{\circ} 44^{\prime} 27.28994^{\prime \prime} \mathrm{E}$ & 0.0018 & 1520.7700 & 0.0026 \\
\hline WNGR & $7^{\circ} 22^{\prime} 19.48065^{\prime \prime} \mathrm{S}$ & 0.0024 & $107^{\circ} 42^{\prime} 52.99020^{\prime \prime} \mathrm{E}$ & 0.0027 & 1271.3211 & 0.0044 \\
\hline
\end{tabular}



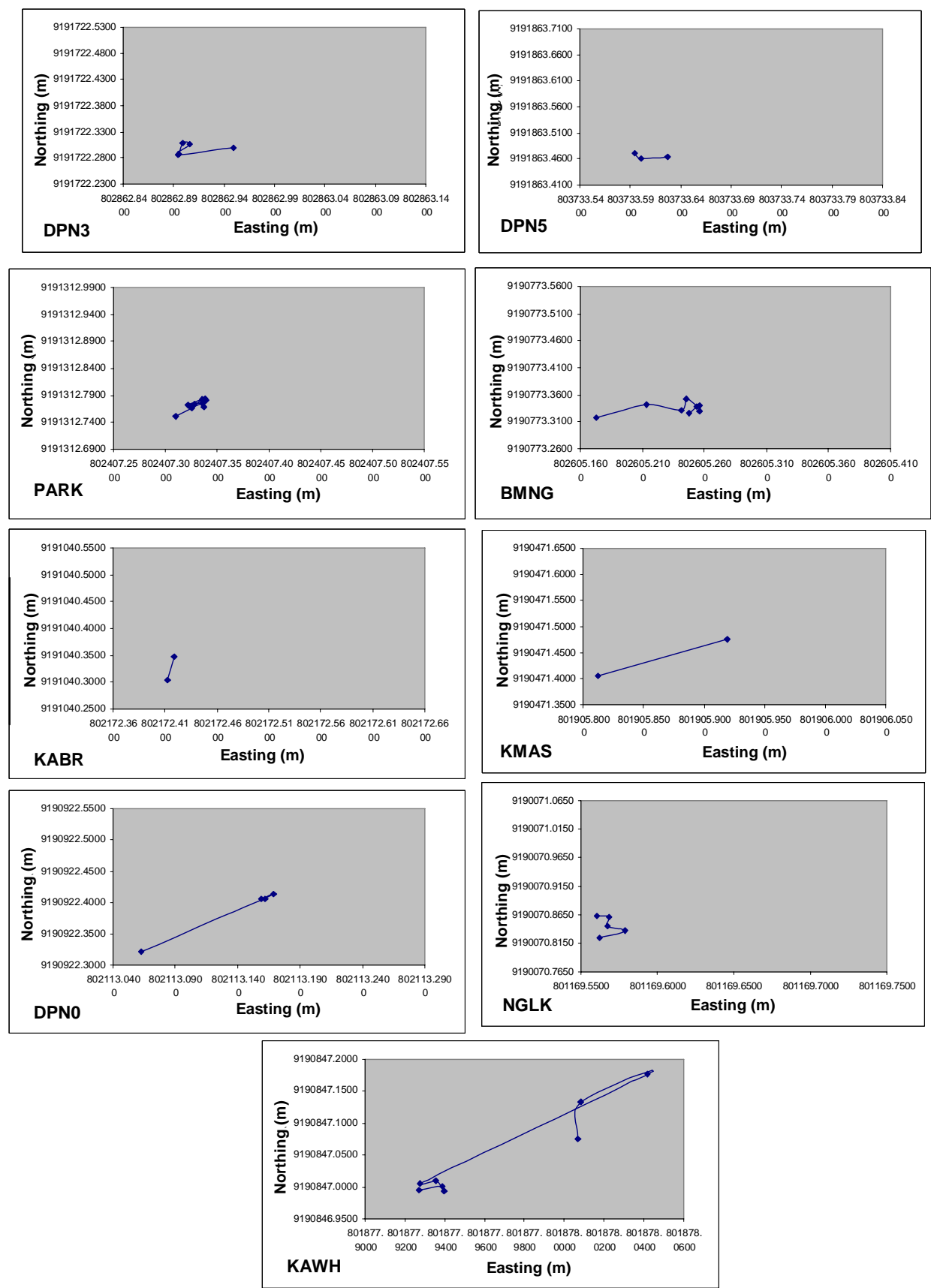

Figure 4 Coordinate changes of GPS points in horizontal directions (campaign 1 - 8). 

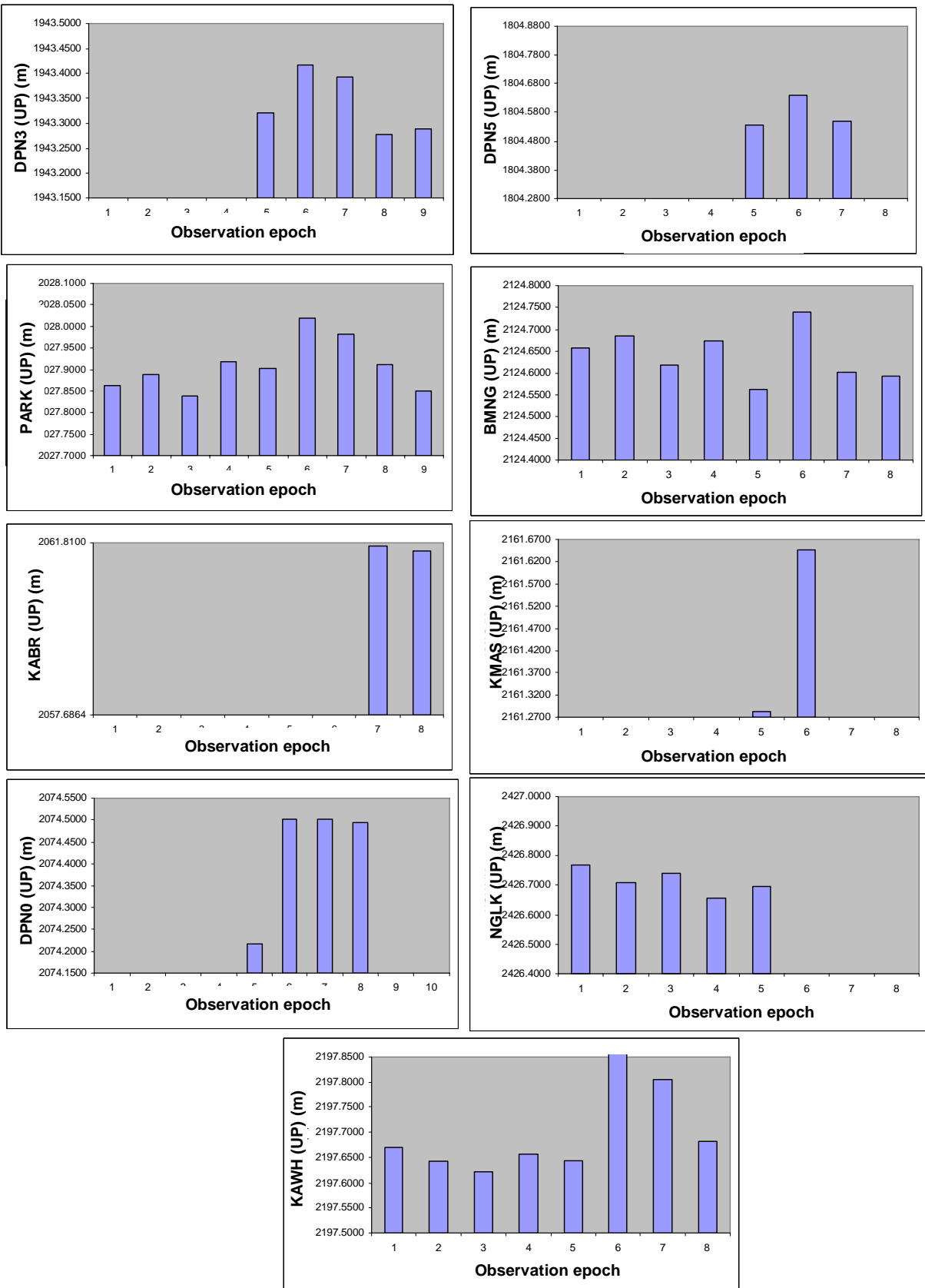

Figure 5 Coordinate changes of GPS points in vertical direction (campaign 1 $-8)$. 

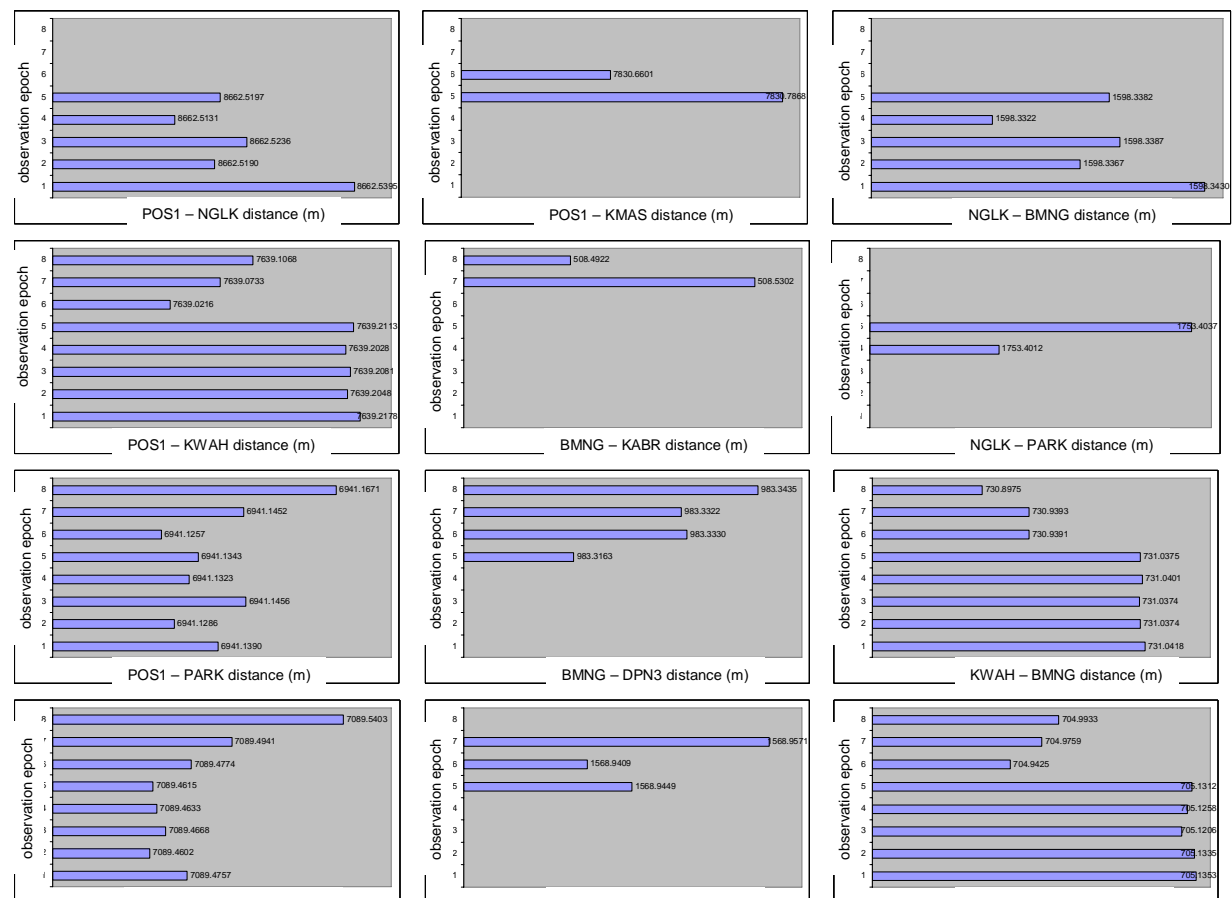

POS1 - BMNG distance (m)

BMNG - DPN5 distance (m)
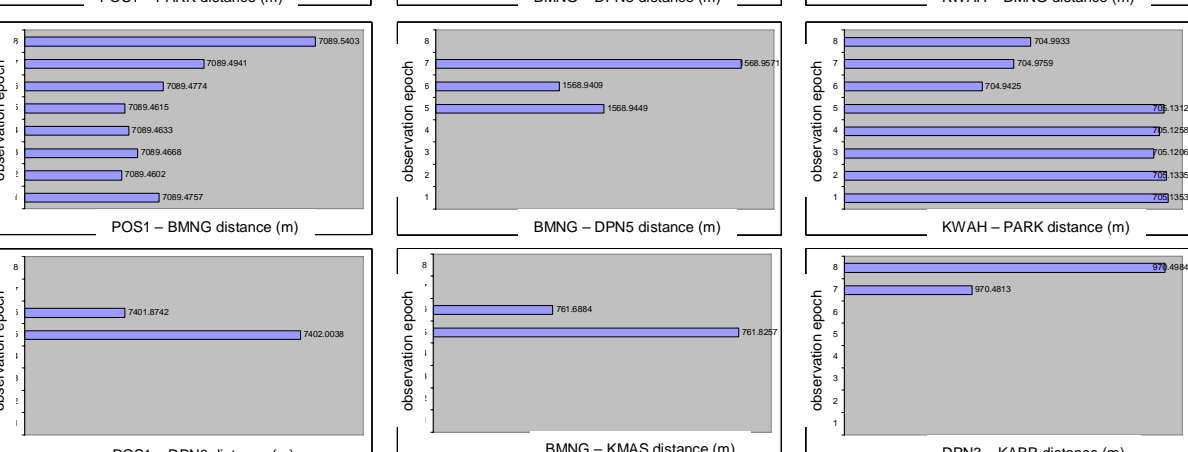

KWAH - PARK distance (m)

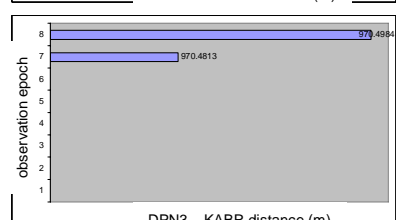

POS1 - DPNO distance (m)

BMNG - KMAS distance (m)

DPN3 - KABR distance $(\mathrm{m})$
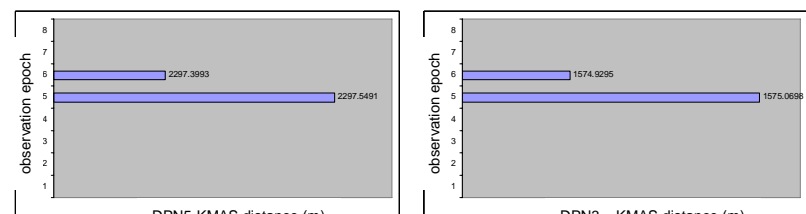

POS1 - DPN3 distance (m)

DPN5-KMAS distance (m)

DPN3 - KMAS distance (m)
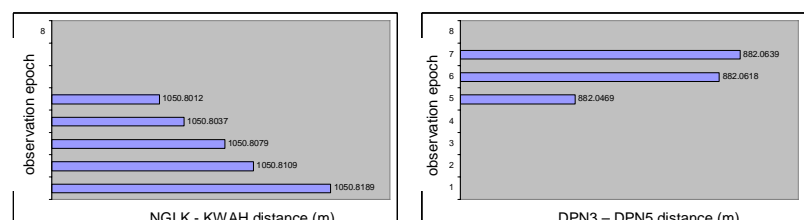

(a)

Figure 6 Baseline changes of GPS point coordinate (campaign 1 - 8). 

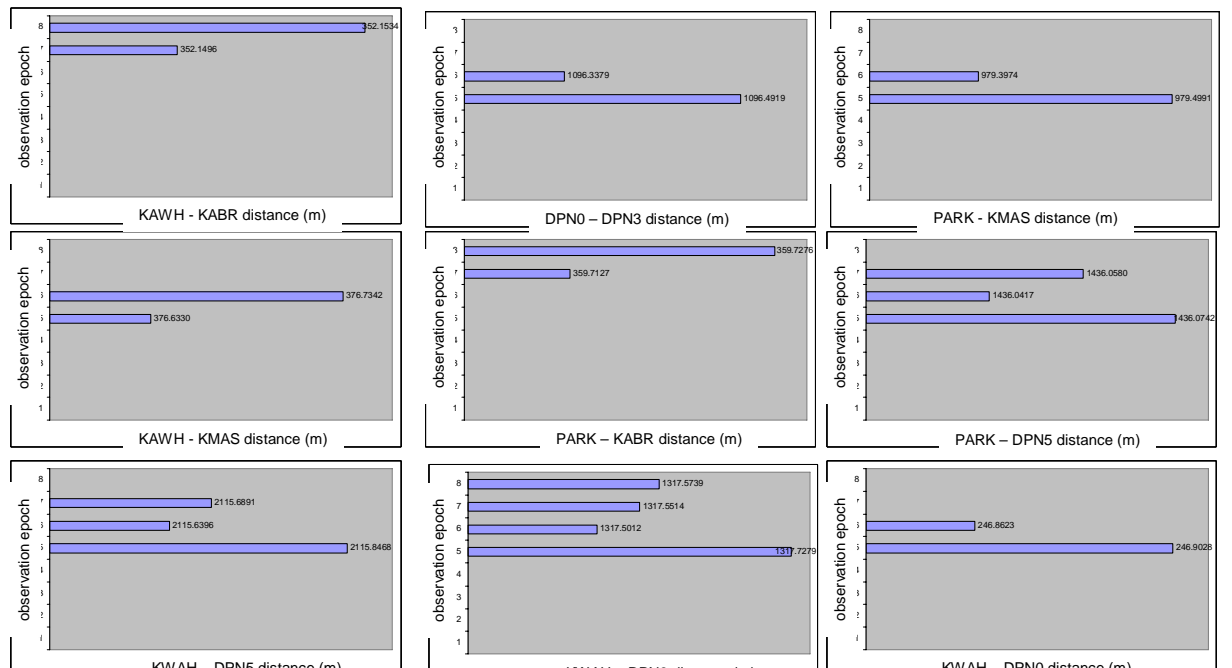

KWAH - DPN5 distance (m)

KWAH - DPN3 distance (m)

KWAH - DPNO distance (m)
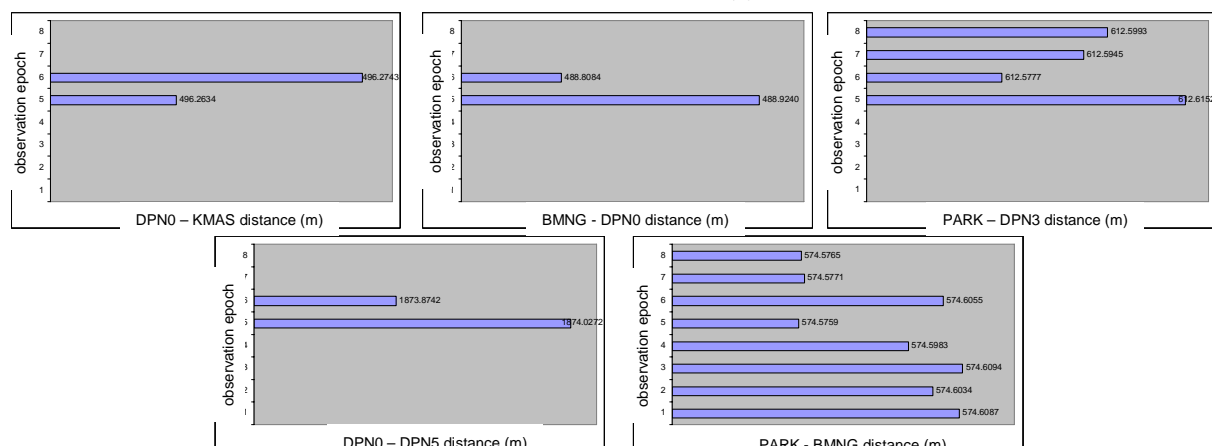

(b)

Figure 6 Baseline changes of GPS coordinate (campaign 1 - 8). (continue)

\subsection{Vector Displacement}

One of the way to understand the deformation pattern in Papandayan volcano by studying vectors movement of each GPS coordinate. Direction of movement of each campaign period separated into horizontal coordinate (easting-northing) Figure 7 and vertical component Figure 8. 
Dina A. Sa rsito, et al.
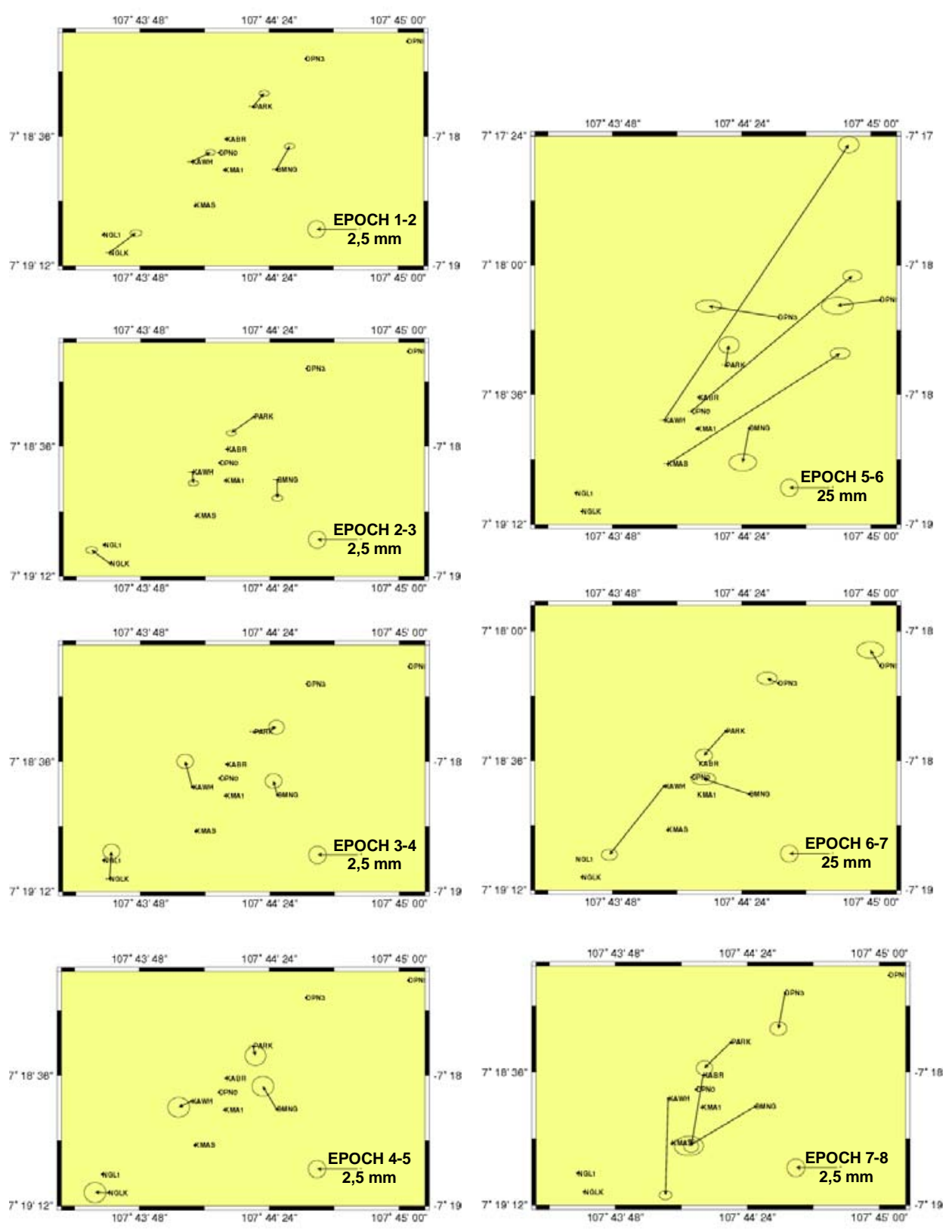

Figure 7 Vector displacement in horizontal direction. 


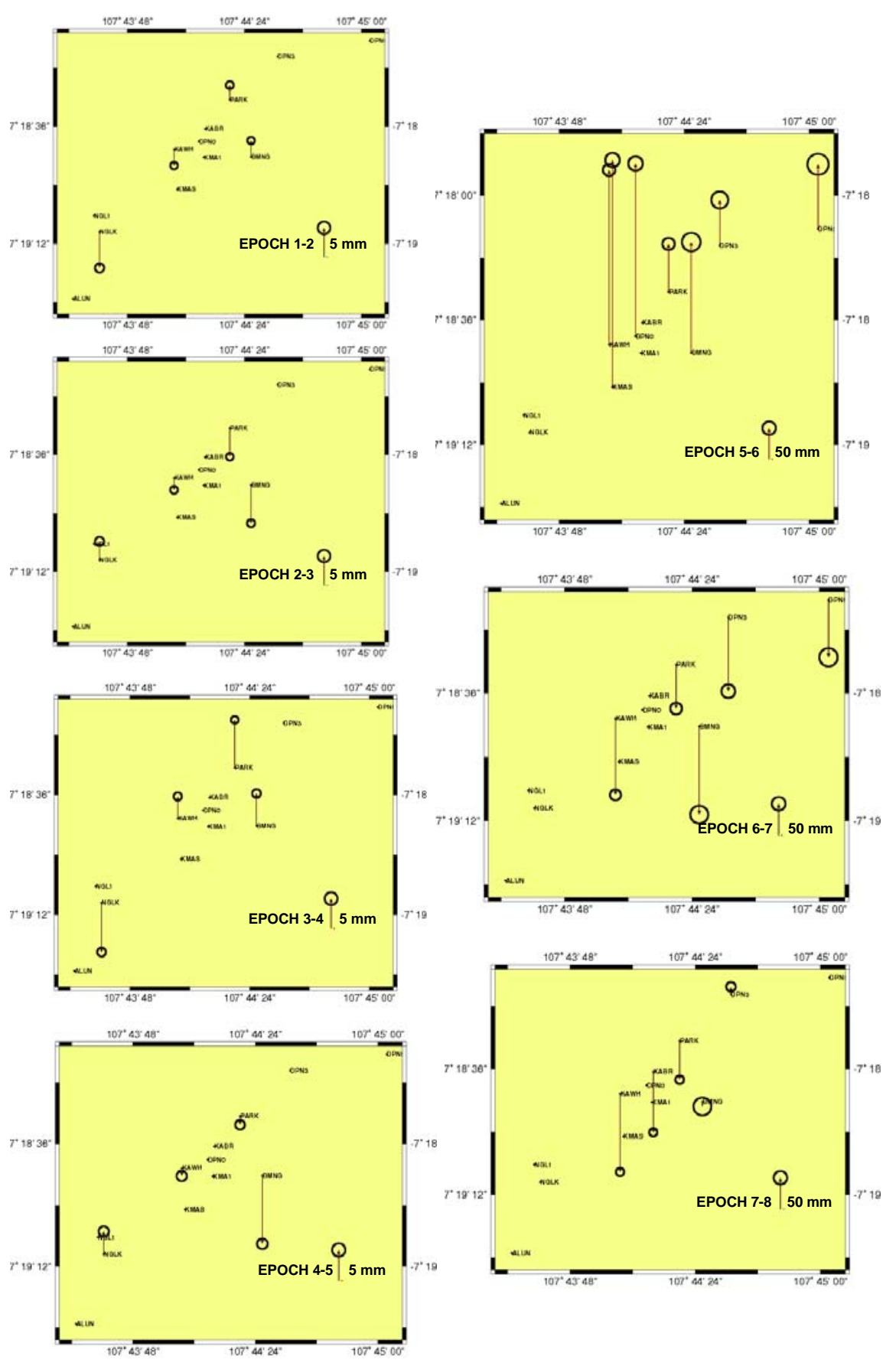

Figure 8 Vector displacement in vertical direction. 


\subsection{Strain Analysis}

In this research, deformation parameters in Papandayan determined by using two 3D resemble models to have detail illustration of deflation and inflation of volcano body comparing by using 2D model. Type A model use the assumption that deformation tensor as a function of translation, dilatation and rotation parameters, while type $\mathrm{B}$, deformation tensor as a function of dilatation and rotation parameters. The principal strain revealed from A and B models calculation show on Figures 9 and 10 Where the orientation pattern of A and B models have the same direction with the orientation of stress and strain which occurred in the volcano, as show on Table 5.

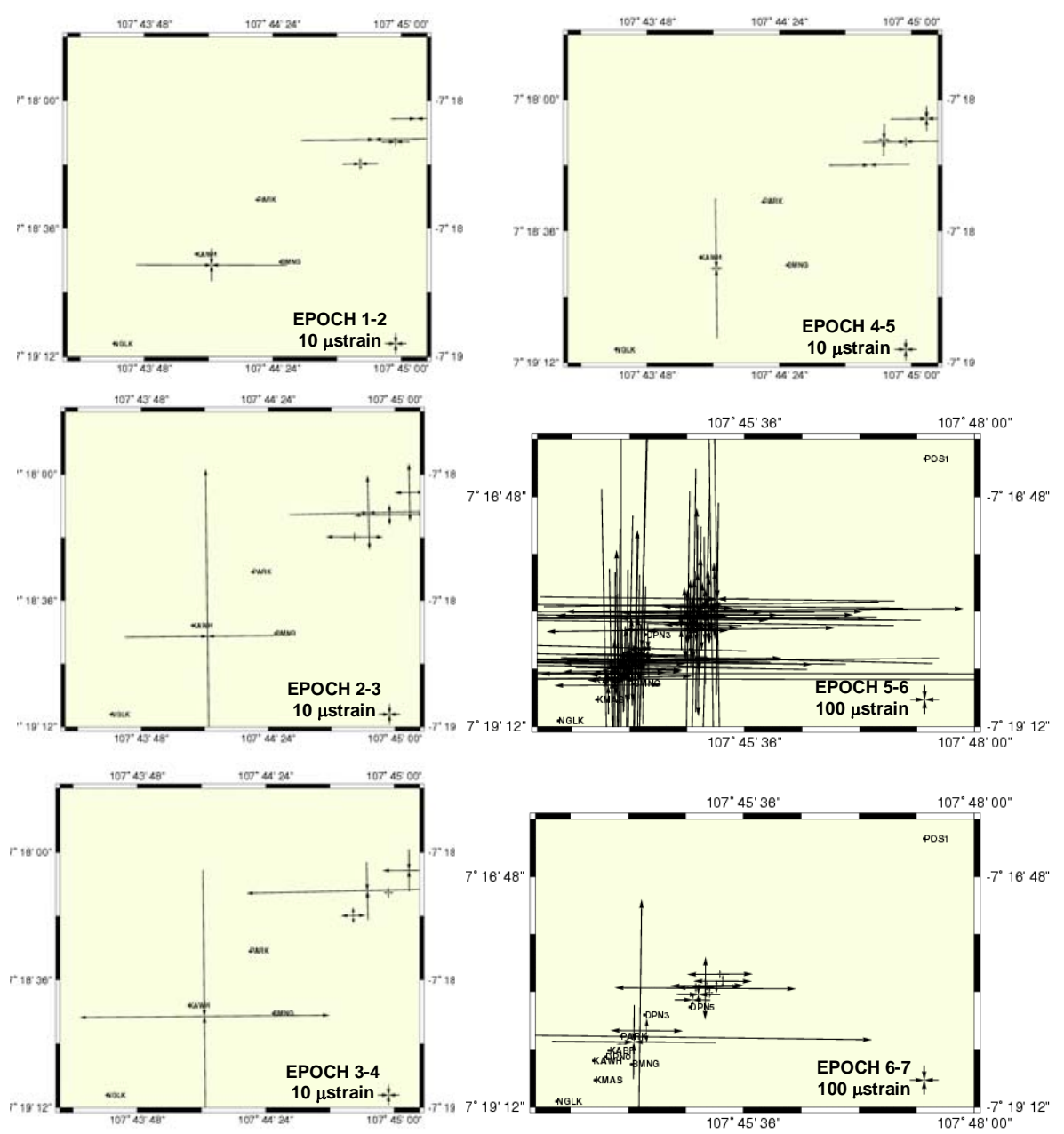

Figure 9 Principal strain orientation by A model. 


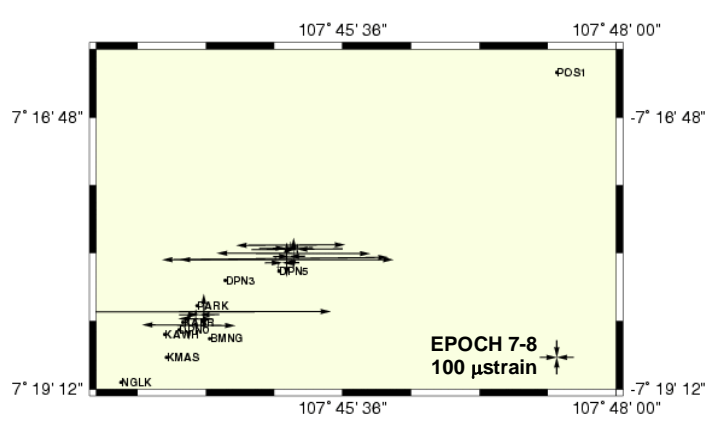

Figure 9 Principal strain orientation by A model. (continue)

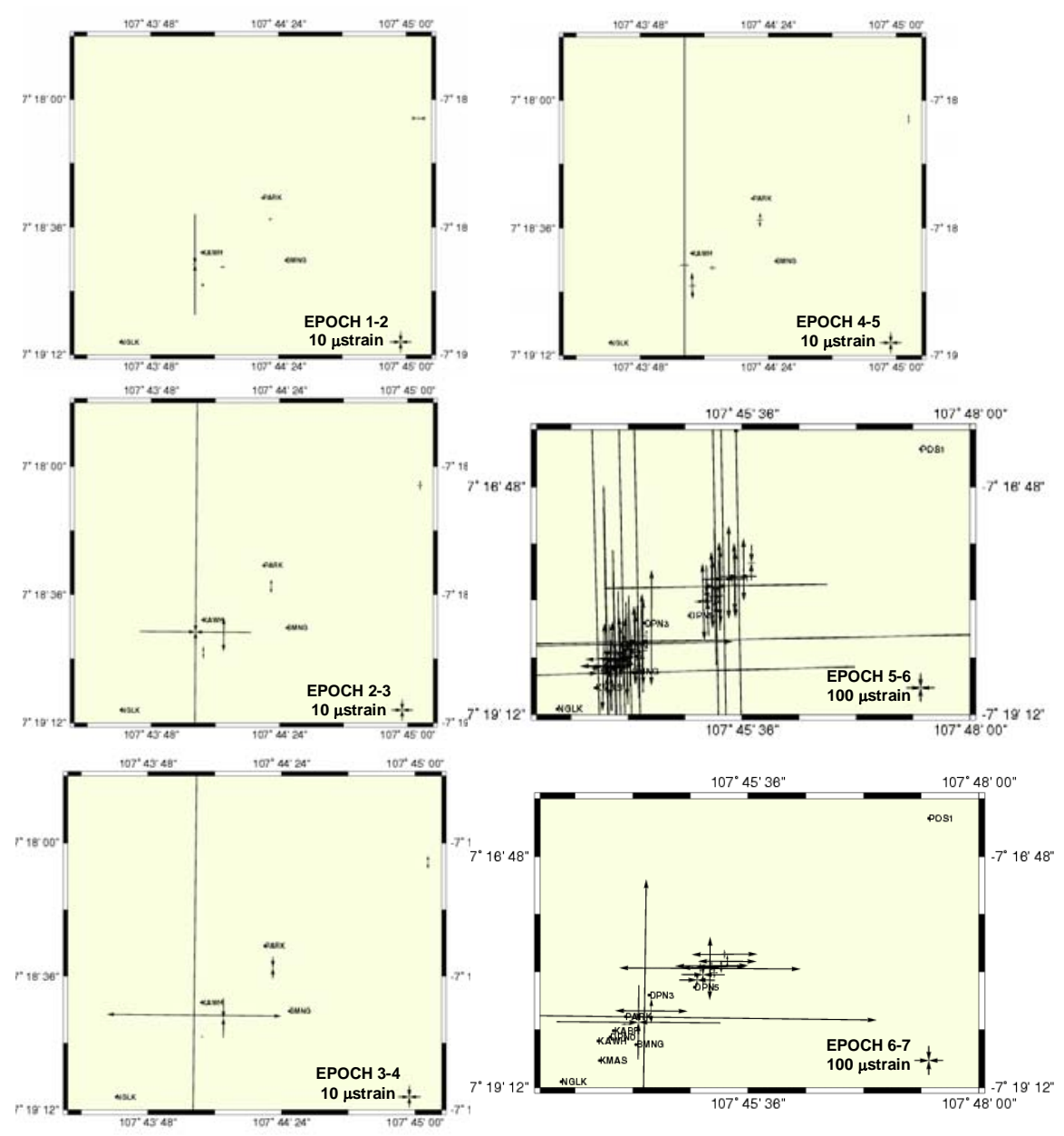

Figure 10 Principal strain orientation by B model. 


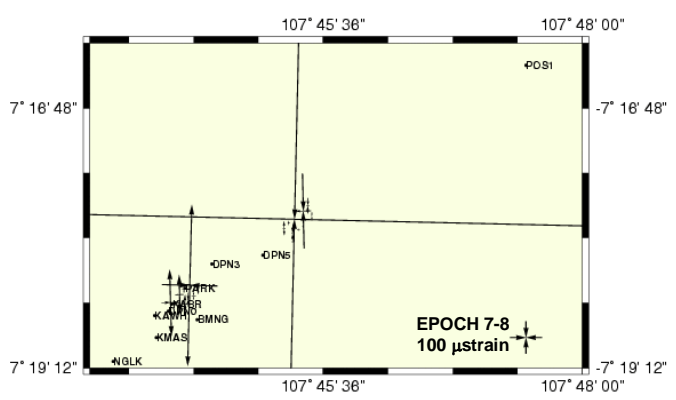

Figure 10 Principal strain orientation by B model. (continue)

Table 5 Orientation and principal strain major of A and B models.

\begin{tabular}{|c|c|c|c|c|c|c|}
\hline \multirow[t]{2}{*}{ Period } & \multicolumn{3}{|c|}{ A model } & \multicolumn{3}{|c|}{ B model } \\
\hline & $\begin{array}{l}\text { extension } \\
\mu \text { strain }\end{array}$ & $\begin{array}{c}\text { contraction } \\
\mu \text { strain }\end{array}$ & $\begin{array}{l}\text { easting axis } \\
\text { orinetation }\end{array}$ & $\begin{array}{c}\text { extension } \\
\mu \text { strain }\end{array}$ & $\begin{array}{c}\text { contraction } \\
\mu \text { strain }\end{array}$ & $\begin{array}{l}\text { easting axis } \\
\text { orinetation }\end{array}$ \\
\hline $1-2$ & -162260 & 10865 & -0.5087 & -0.2399 & -1.6277 & 0.7472 \\
\hline $2-3$ & 273930 & 11.4691 & -0.0780 & -1.2343 & 10.9018 & -1.0004 \\
\hline $3-4$ & 110430 & 46747 & 0.8764 & -0.5707 & 2.7209 & -1.4289 \\
\hline $4-5$ & -36.7200 & 9.7049 & -0.9549 & -0.9598 & 23.8483 & -0.3095 \\
\hline $5-6$ & 948630 & -14.7688 & -0.8617 & 4.8651 & -31.6804 & -1.2367 \\
\hline $6-7$ & 72.7020 & 293542 & 0.5590 & -3.5472 & 20.9990 & -1.2990 \\
\hline $7-8$ & 212480 & -12.1843 & 0.4964 & -1.8166 & -0.4951 & 1.1694 \\
\hline
\end{tabular}

\subsection{Seismic Analysis}

Analysis of seismic data carried out from 1998 to 2005, where a strong increasing occurred in November 2002 following by the eruptions, Figure 11. During 2000 to 2005 Papandayan volcano dominated by B type volcanic earthquake, Figure 12. A large energy accumulation occurred during the period of \#5 to \#6 campaign, the increasing occurred three months long, as $2.51 \mathrm{E}+15$ erg, where the eruption happen, Figure 13. The hypocenter undetermined caused of less data, determination hypocenter show on Figure 14.
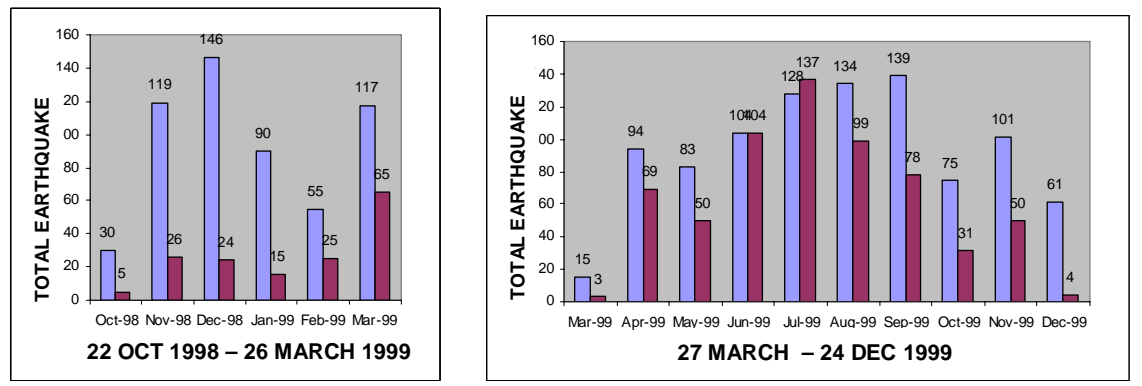

Figure 11 Statistics of tectonic and volcanic earthquake during October 1998 to August 2005. 

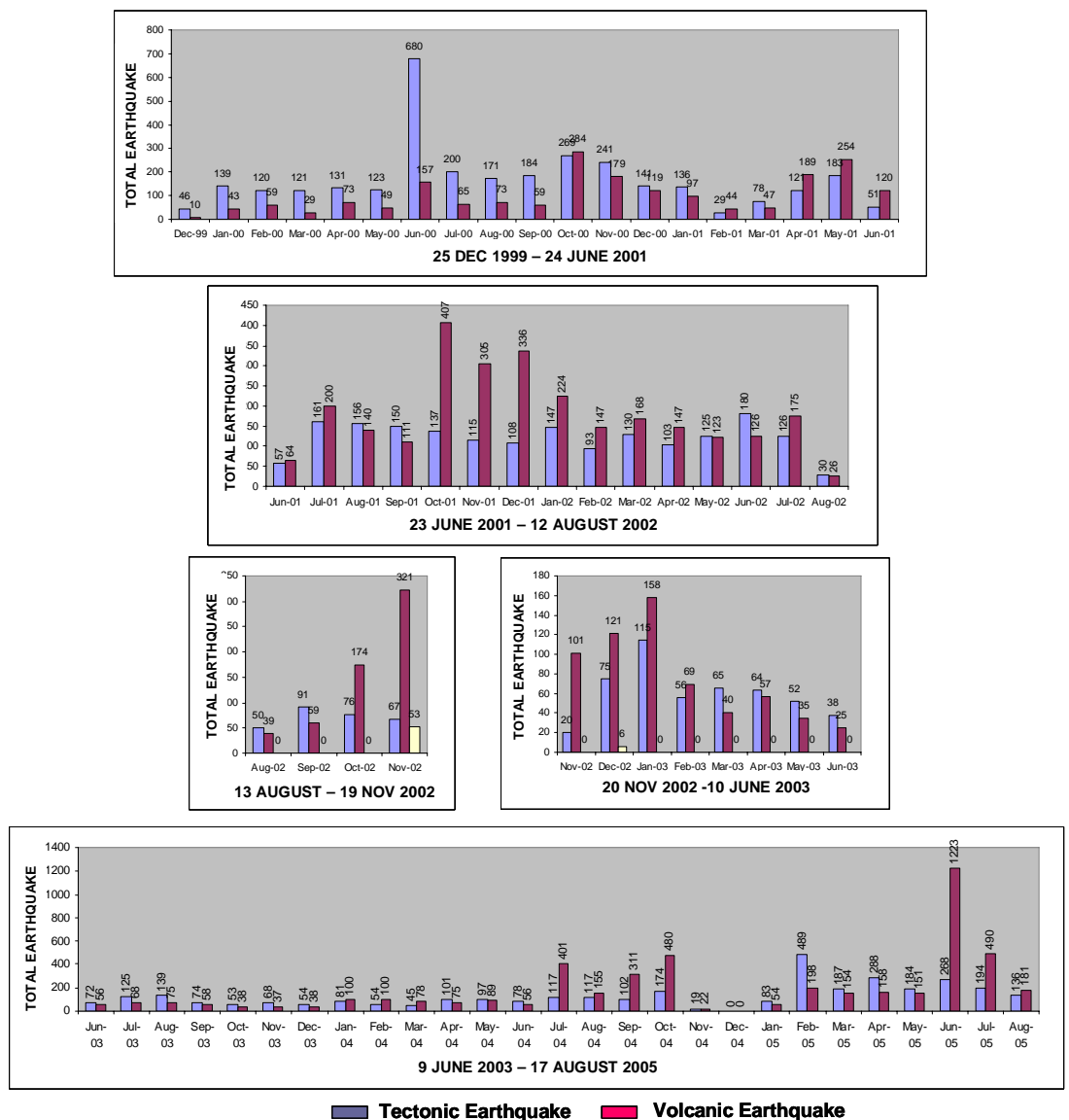

Figure 11 Statistics of tectonic and volcanic earthquake during October 1998 to August 2005. (continue)

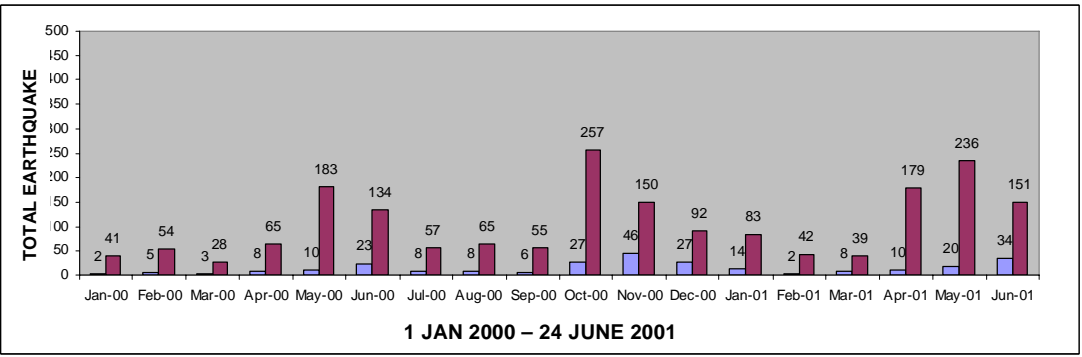

Figure 12 Statistics of Volcanic A and B during October 1998 to August 2005. 


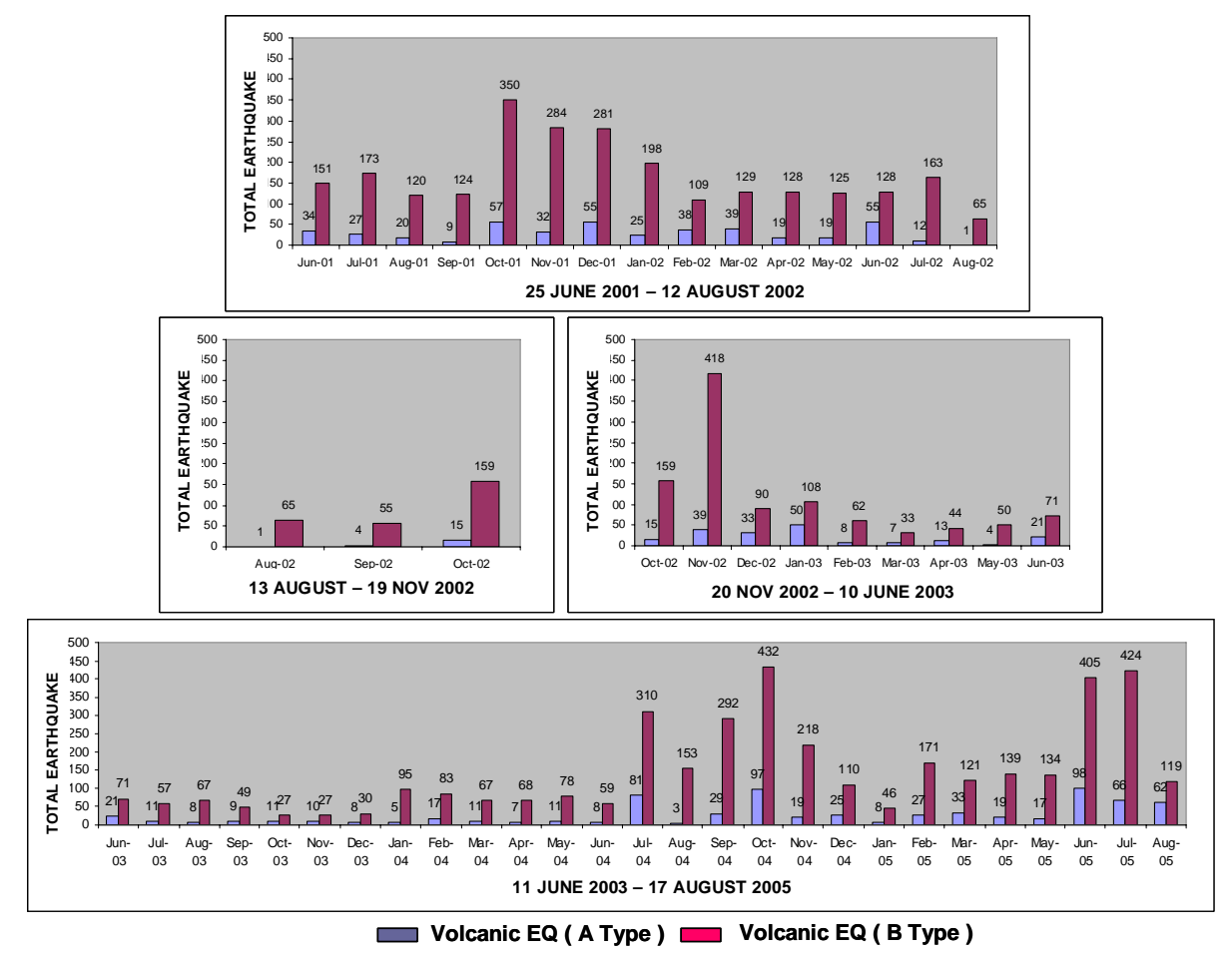

Figure 13 Statistics of Volcanic A and B during October 1998 to August 2005. (continue)

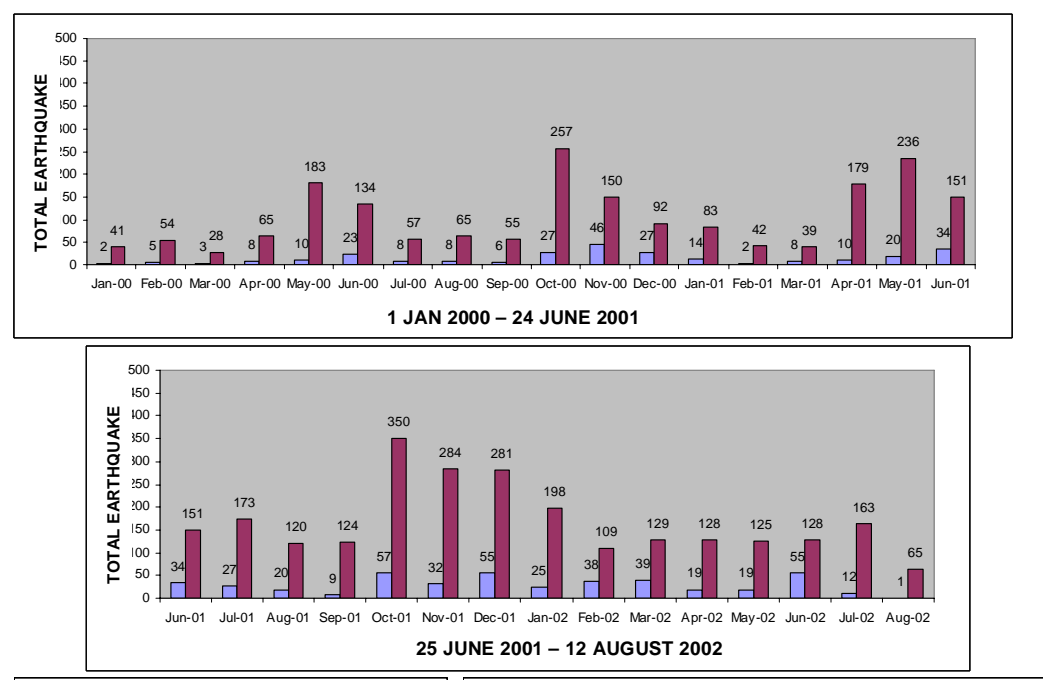

Figure 14 Statistics of volcanic energy accumulation during January 2000 to August 2005 


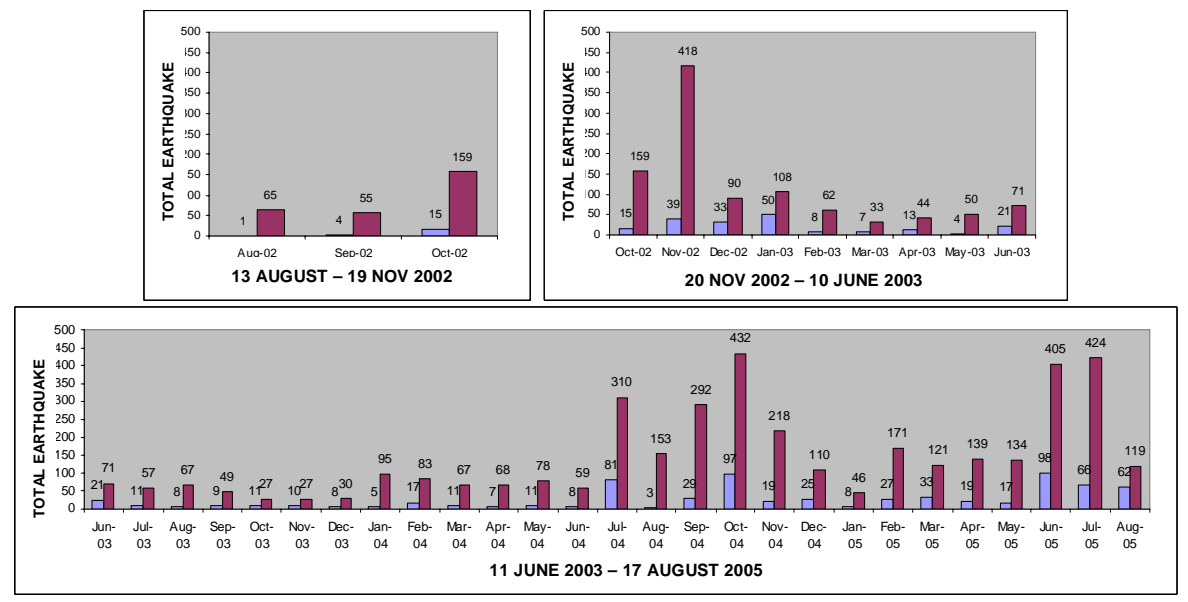

$\square$ Volcanic EQ ( A Type ) Volcanic EQ ( B Type )

Figure 14 Statistics of volcanic energy accumulation during January 2000 to August 2005. (continue)
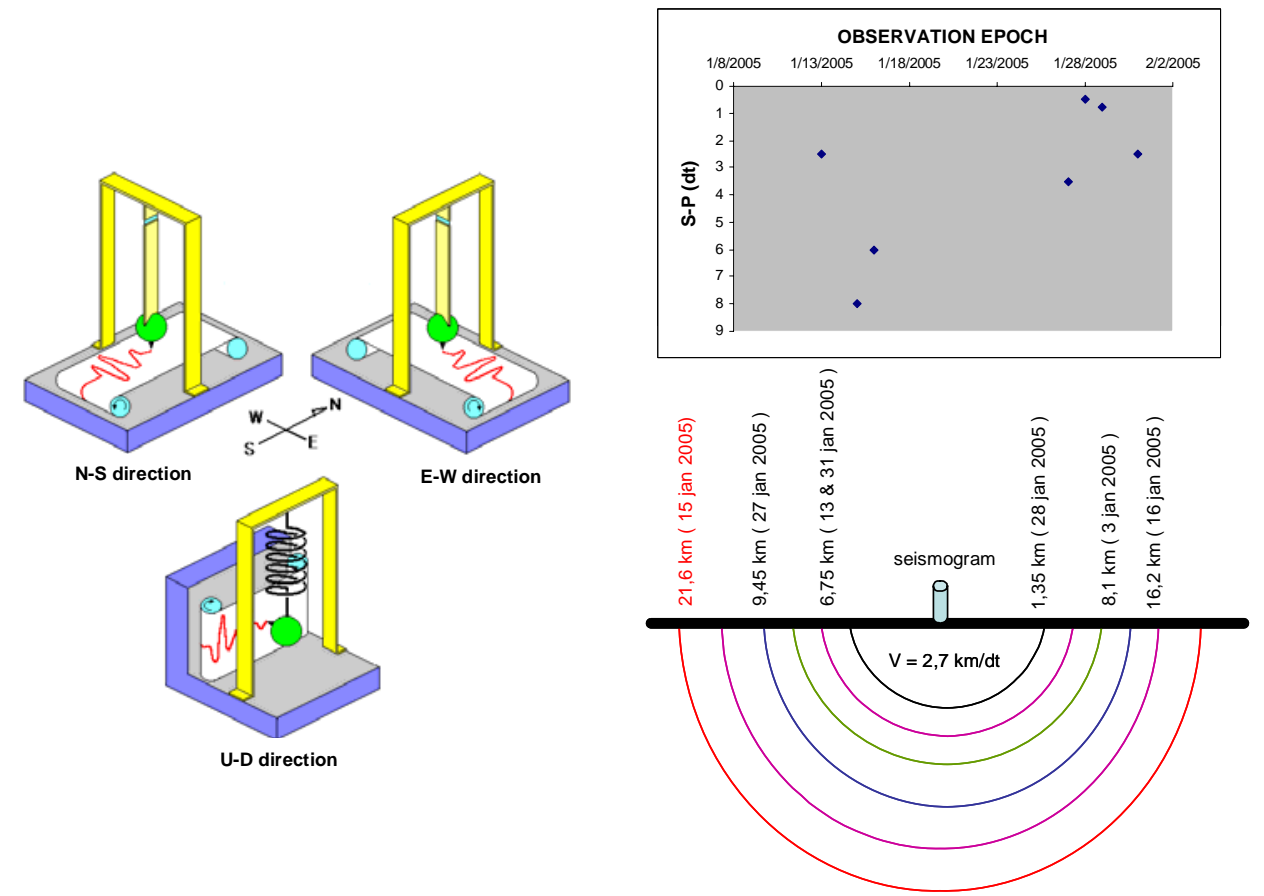

Figure 15 Ambiguities hypocenters determination in one seismogram with one component, in period January 2005. 


\subsection{Correlation of Deformation Analysis and Seismicity Pattern in Papandayan Volcano}

\section{Period 1-2}

Period 1-2, the whole direction of horizontal vectors movement (NGLK, BMNG, KAWH, PARK) are to the northeast. Based on geometric movement pattern estimated the stress source come from the southwest that pushed to the northeast. Movement pattern of BMNG bigger compare to PARK, this means by different rheology. No significant correlation. This period estimate there is an inflation in volcano body caused by magma pressure which moved to KAWH zone in the northeast.

\section{Period 2-3}

Period 2-3, entirely movement BMNG and PARK are bigger compare to NGLK and KAWH. Based on geometric pattern, showed shrinking of pressure source. No significant correlation. This period estimate deflation of volcano body caused of decreasing of pressure source, the source move to NGLK zone.

\section{Period 3-4}

Period 3-4, entirely baseline became shrinking, NGLK move to the north. No significant correlation. This period estimate inflation of volcano body caused of pressure source, the source move to the northeast (KAWH zone).

\section{Period 4-5}

Period 4-5, entirely almost similar with period 2-3, shrinking of BMNG much bigger than KAWH and PARK. During this period accumulation of seismic energy record $1.47 \mathrm{E}+16$ erg with the average events of volcanic earthquake 193 per month, where during this period there are 1911 B type event of volcanic earthquake. No significant correlation because there is no GPS data when seismicities increase.

\section{Period 5-6}

Period 5-6, eruption started during this period, entirely horizontal deformation extremely occurred with northeast direction, which has large value on the crater part than getting smaller to POS1 direction. While vertical movement increase at all points, with large value than getting smaller to POS1 direction. Detail analysis of this period GPS data has been done by Abidin (2003). In this period the accumulation of seismic energy around $2.51 \mathrm{E}+15$ erg during three months with total volcanic B type 279 (total volcanic earthquake 593). This accumulation energy is very big and caused big deformation. During this period the eruption occurred. 


\section{Period 6-7}

Period 6-7, movement direction both vertical and horizontal became deflate with southwest direction. BMNG-KAWH baseline became shorter. Seismic activities during this period decreased compare to former campaign. In this period estimate decreasing of pressure source, the source moved to the southwest, deflation.

\section{Period 7-8}

Period 7-8, small changes of move direction both horizontal and vertical compare to period 6-7, keep moving to southwest direction and decrease. Small changes of baseline compare to period 6-7. Averagely, geometric pattern showed the occurrence of deflation, anyway not continuous deflation from period 6-7 except new deflation pattern caused by the extremely accumulation of seismic energy during October 2004 and June 2005, where during this period volcanic activity increase, which occurred before the \#8 campaign carried out. On \#8 campaign estimated the decreasing of pressure source which caused deflation of volcano body.

\subsection{Pressure Source Analysis (Mogi Model)}

This model [4], assumption earth crust as half elastic medium and deformation which occur caused by pressure source as magma ball embedded in a certain medium depth. If the hydrostatic pressures of the ball changes, then caused symmetrical deformation of the surface. Pressure source determinations show on Figure 17.

The estimation of pressure source derived from all GPS campaigns, showed different of location of pressure source. Entirely, estimation of pressure source which derived from NGLK, KMAS, BMNG, KAWH and PARK are located around the southwest part at 1000 depth beneath NGLK and KAWH (1300.067 $\mathrm{m}$ above ellipsoid). An anomaly showed on period 1 to 2 compare to next period, where the source location too far to the northwest. This condition may caused by distribution of geometric network and measurement data was not long enough. The total seismic energy accumulation was unknown because less data.

The pressure source of the first group is the result of period 2-3, 3-4 and 4-5 have same zone to KAWH with 1000 depth. Energy accumulation on period 2-3 and some of period 3-4 (data begin on January 1, 2000) unknown exactly. While period 4-5 with total accumulation energy $1.47 \mathrm{E}+16$ erg show the pressure source start to close to KAWH. The pressure source of the second group on period 5-6 located in the same horizontal zone with the first group 
except depth different, that is $1000 \mathrm{~m}$ depth. On this period, characteristic of seismicity was very high, that is during the eruption period. The pressure source of the third group period 6-7 and 7-8 which is showed approach KAWH with $1000 \mathrm{~m}$ depth.

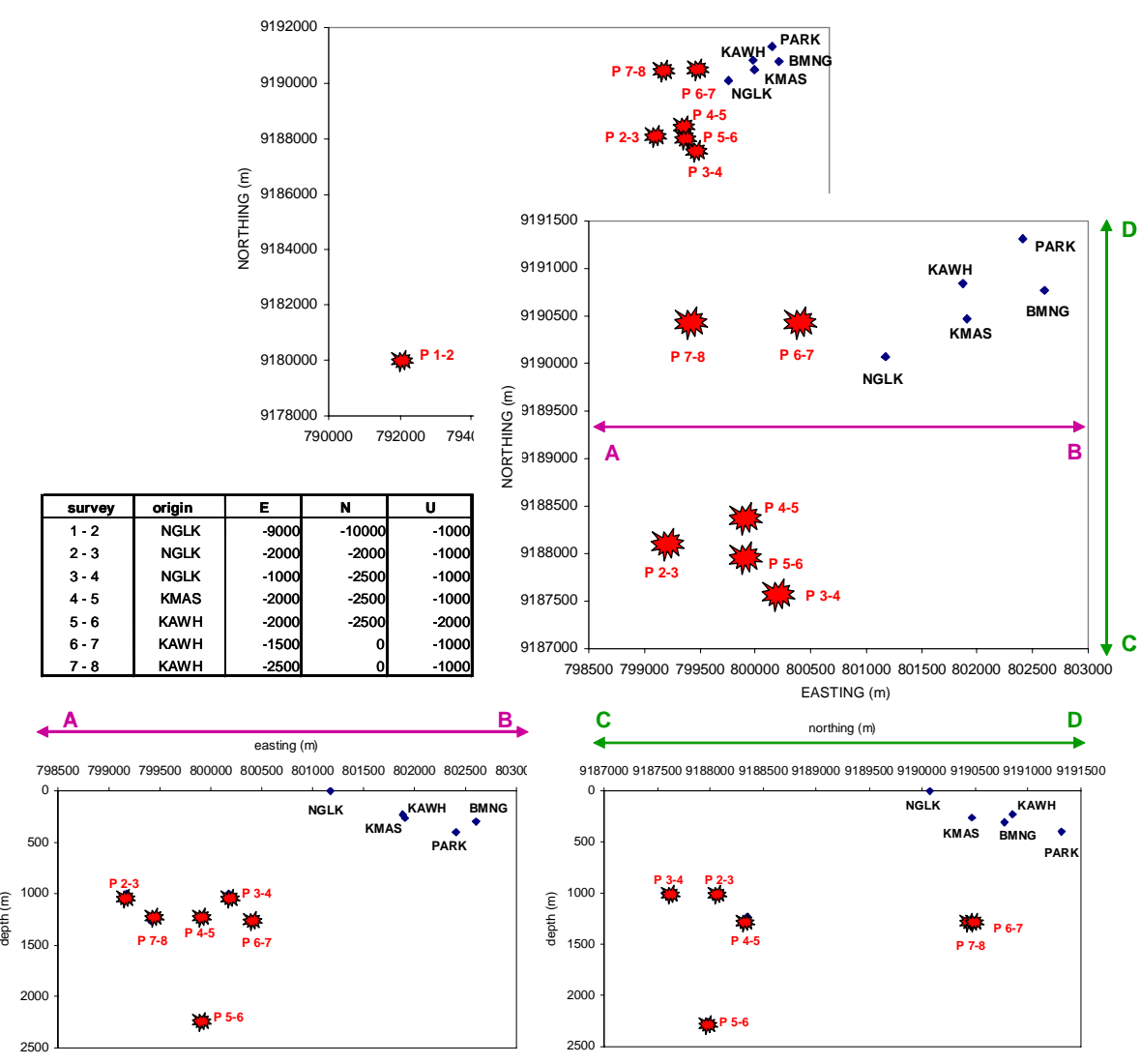

Figure 16 Pressure source determinations by Mogi model, derived from period of measurement 1 to 8 .

\section{$5 \quad$ Closing Remark}

Conclusion:

1. GPS survey capable for volcano deformation study with high precision (mm) relatively. Even to have this precision is not an easy thing. To reach $\mathrm{mm}$ precision needs Geodetics receiver dual-frequency, detail survey planning, strictly measurement strategy and data processing strategy by 
using scientific software. Because of high capability for deformation signal, then volcano deformation study by using GPS survey method will continue.

2. Ambiguity occurred when determined hypocenter if only use one component seismogram.

3. Correlation between determination result of deformation by using GPS data and seismic characteristic of Papandayan volcano.

\begin{tabular}{|c|c|c|c|}
\hline Seismic data & $\begin{array}{c}\text { Frequency of A and } \\
\text { B volcanic } \\
\text { earthquake }\end{array}$ & Magnitude & $\begin{array}{c}\text { Energy } \\
\text { accumulation }\end{array}$ \\
\hline Vector displacement & + & + & + \\
\hline Baseline Changes & + & + & + \\
\hline Principal strain & + & + & + \\
\hline $\begin{array}{c}\text { Pressure source } \\
\text { Analysis }\end{array}$ & + & + & + \\
\hline
\end{tabular}

4. Correlation between geodetic aspect with geologic and geophysics aspect in Papandayan volcano

\begin{tabular}{|l|l|}
\hline \multicolumn{1}{|c|}{ Geodetics Aspect (geometric) } & \multicolumn{1}{c|}{ Geologic/geophysics Aspect } \\
\hline $\begin{array}{l}\text { Preference pattern of vector displacement } \\
\text { to the northeast direction }\end{array}$ & $\begin{array}{l}\text { Pressure source tend to growth to northeast } \\
\text { direction }\end{array}$ \\
\hline $\begin{array}{l}\text { Alternating changes of vector direction in } \\
\text { both horizontal and vertical components } \\
\text { nearly similar in certain period }\end{array}$ & $\begin{array}{l}\text { The growth up of pressure source very } \\
\text { fluctuate caused inflation when the activity } \\
\text { increased or deflation when decreased }\end{array}$ \\
\hline $\begin{array}{l}\text { Large principal strain component in } \\
\text { crater zone which getting smaller close to } \\
\text { POS1 }\end{array}$ & $\begin{array}{l}\text { There are forces which pushed the crater } \\
\text { flank }\end{array}$ \\
\hline $\begin{array}{l}\text { Pattern principal extension component } \\
\text { direct to the northeast }\end{array}$ & $\begin{array}{l}\text { The growth up of pressure source seem to } \\
\text { northeast direction }\end{array}$ \\
\hline
\end{tabular}

5. Based on deformation value and accumulation of seismic energy, then whole body of Papandayan is elastic, where inflation occurred when energy accumulated and deflation when low accumulation energy.

Suggestion:

1. Necessary more installation of multi-components seismic station to determined the hypocenter which needs for prediction of magma body.

2. To more understand the correlation of deformation and seismic energy, necessary. Continuous geometric monitoring together with seismic monitoring.

\section{References}

[1] Abidin, H.Z., Andreas, H., Gamal, M., Suganda, O.K., Meilano, I., Hendrasto, M., Kusuma, M.A., Darmawan, D., Purbawinata, M.A., Wirakusumah, A.D. \& Kimata, F., Ground Deformation During 
Papandayan Volcano 2002 Eruption As Detected By GPS Surveys, PROC.ITB Eng. Science Vol.35 B, No.1, pp 45-60 (2003).

[2] Beutler, G., Bock, H., Brockmann, E., Dach, R., Fridez, P., Gurtner, W., Hugentobler, U., Ineichen, D., Johnson, J., Meindl, M., Mervant, L., Rothacher, M., Schaer, S., Springer, T., Weber, R., Bernese GPS software version 4.2, U. Hugentobler, S. Schaer, P. Fridez (Eds.), Astronomical Institute, University of Berne, 515 pp (2001).

[3] VSI, Situs internet dari Direktorat Vulkanologi dan Mitigasi Bencana Geologi (DVMBG), alamat http://www.vsi.esdm.go.id/, 25 September (2005).

[4] Mogi, K., Relations Between The Eruptions of Various Volcanoes and The Deformation of The Ground Surfaces Around Them, Bulletin of Earthquake Research Institute University of Tokyo, Vol. 36, pp. 99-134 (1958). 